\title{
Identification of Natural Compounds (Proanthocyanidin and Rhapontin) as High-Affinity Inhibitor of SARS-CoV-2 Mpro and PLpro using Computational Strategies
}

\author{
Type
}

Research paper

\section{Keywords}

natural compounds, COVID-19, SARS-CoV-2, Mpro, Molecular docking and Simulation, 3CLpro, PLpro

\begin{abstract}
Introduction

The emergence of a new and highly pathogenic coronavirus (SARS-CoV-2) in Wuhan (China) and its spread worldwide has resulted in enormous social and economic loss. Amongst many proteins encoded by SARS-CoV-2 genome, the main protease (Mpro) or chymotrypsin-like cysteine protease (3CLpro) and Papain-like protease (PLpro) serve as an attractive drug target.
\end{abstract}

\section{Material and methods}

We screened a library of 2267 natural compounds against Mpro and PLpro using high throughput virtual screening (HTVS). 50 top-scoring compounds against each protein in HTVS were further evaluated by standard-precision (SP) docking. Compounds with SP docking energy of $\leq-8.0 \mathrm{kcal}$ mol-1 against Mpro and $\leq-5.0 \mathrm{kcal}$ mol-1 against PLpro were subjected to extra-precision (XP) docking. Finally, six compounds against each target proteins were identified and subjected to Prime/MM-GBSA free energy calculations. Compounds with the lowest Prime/MM-GBSA energy were subjected to molecular dynamics simulation to evaluate the stability of protein-ligand complexes.

\section{Results}

Proanthocyanidin and Rhapontin were identified as the most potent inhibitors of Mpro and PLpro, respectively. Analysis of protein-inhibitor interaction revealed that both protein-inhibitor complexes were stabilized by hydrogen bonding and hydrophobic interactions. Proanthocyanidin interacted with the catalytic residues (His41 and Cys145) of Mpro, while Rhapontin contacted the active site residues (Trp106, His272, Asp286) of PLpro. The docking energies of Proanthocyanidin and Rhapontin towards their respective targets were -10.566 and $-10.022 \mathrm{kcal} / \mathrm{mol}$.

\section{Conclusions}

This study's outcome may serve Proanthocyanidin and Rhapontin as a scaffold to build more potent inhibitors with desirable drug-like properties. However, it requires further validations by in vitro and in vivo studies. 


\section{$1 \quad$ Abstract}

2 Introduction: The emergence of a new and highly pathogenic coronavirus (SARS-CoV-2) in

3 Wuhan (China) and its spread worldwide has resulted in enormous social and economic loss.

4 Amongst many proteins encoded by SARS-CoV-2 genome, the main protease (Mpro) or

5 chymotrypsin-like cysteine protease (3CLpro) and Papain-like protease (PLpro) serve as an

6 attractive drug target.

7 Material and Methods: We screened a library of 2267 natural compounds against Mpro and

8 PLpro using high throughput virtual screening (HTVS). 50 top-scoring compounds against

9 each protein in HTVS were further evaluated by standard-precision (SP) docking. Compounds

10 with SP docking energy of $\leq-8.0 \mathrm{kcal} \mathrm{mol}^{-1}$ against Mpro and $\leq-5.0 \mathrm{kcal} \mathrm{mol}^{-1}$ against PLpro

11 were subjected to extra-precision (XP) docking. Finally, six compounds against each target

12 proteins were identified and subjected to Prime/MM-GBSA free energy calculations.

13 Compounds with the lowest Prime/MM-GBSA energy were subjected to molecular dynamics

14 simulation to evaluate the stability of protein-ligand complexes.

15 Results: Proanthocyanidin and Rhapontin were identified as the most potent inhibitors of

16 Mpro and PLpro, respectively. Analysis of protein-inhibitor interaction revealed that both

17 protein-inhibitor complexes were stabilized by hydrogen bonding and hydrophobic interactions. Proanthocyanidin interacted with the catalytic residues (His41 and Cys145) of

19 Mpro, while Rhapontin contacted the active site residues (Trp106, His272, Asp286) of PLpro.

20 The docking energies of Proanthocyanidin and Rhapontin towards their respective targets

21 were -10.566 and $-10.022 \mathrm{kcal} / \mathrm{mol}$. 
22 Conclusions: This study's outcome may serve Proanthocyanidin and Rhapontin as a scaffold

23 to build more potent inhibitors with desirable drug-like properties. However, it requires

24 further validations by in vitro and in vivo studies.

25 Keywords: COVID-19; Natural compounds; Molecular docking and Simulation; Mpro;

26 3CLpro; PLpro.

27

28

29

30

31

32

33

34

35

\section{Introduction}

Coronaviruses (CoVs) are enveloped, positive-sense single-stranded RNA virus belonging to the Coronaviridae family of the order Nidovirales. CoVs have been broadly classified into four genera, i.e., $\alpha-, \beta-, \gamma$ - and $\delta$-CoVs [1]. CoVs can trigger moderate to severe upper respiratory tract and gastrointestinal infections in mammals, birds, and humans. Currently, six human coronaviruses (HCoV), namely HCoV-229E, HCoV-OC43, HCoVNL63, HCoV-HKU1, severe acute respiratory syndrome-CoV (SARS-CoV), middle east respiratory syndrome (MERS-CoV), have been reported to cause common cold and pneumonia-like infection. Recently, a seventh member of HCoV, i.e., SARS-CoV-2 (COVID19) has been reported in the Wuhan city (Hubei province, China) to cause severe pneumonialike infection [2]. Since then, SARS-CoV-2 has spread throughout the world and has caused more than 79 million confirmed cases of COVID-19, including more than 1.75 million deaths (https://covid19.who.int; accessed on Dec 27, 2020). Out of the seven HCoVs, three (SARS-

CoV, SARS-CoV-2, and MERS-CoV) are highly virulent and cause a severe respiratory infection, while the other four HCoVs (HCoV-229E, HCoV-OC43, HCoV-NL63, and HCoVHKU1) are mild and cause only common colds-like symptoms. 
The genome of SARS-CoV-2 is $29.9 \mathrm{~kb}$ in length, consisting of flanking untranslated

45 regions (UTRs) at both $5^{\prime}$ and $3^{`}$ ends. The viral genome also possesses 11 open reading

46 frames (ORFs); ORF 1a and 1b present at $5^{`}$ proximal RNA genome encodes for polyprotein

47 and constitute nearly $70 \%$ of the genome [3,4]. ORF1a and $1 \mathrm{~b}$, which are expressed as

48 polypeptides ppla and pplab respectively, undergo proteolytic cleavage to produce 16

49 putative nonstructural proteins (nsps) [5]. The other end of the viral genome, i.e., 3` end,

50 comprises 12 nested ORFs, which ultimately code for structural proteins i.e., spike (S),

51 envelope (E), membrane (M), nucleocapsid (N), and other accessory proteins. Out of these

52 virus proteins, the main protease (Mpro), also known as chymotrypsin-like cysteine protease

53 (3CLpro) encoded by nsp 5 has been established to play a significant role in viral gene

54 expression, replication and thus appears to be an exciting target for SARS-CoV-2 drug design

55 [6]. Another protease of SARS-CoV-2 known as PLpro has been comprehensively studied

56 recently due to its involvement in the process of viral replication and host immune

57 modulation. The principal function of PLpro and 3CLpro is to process the viral polyprotein in

58 an organized manner, where PLpro has supplementary function of shedding ubiquitin and

59 ISG15 tags from host cell proteins, thus assisting the virus to escape host innate immune

60 responses [7]. Hence, lately, it was contended that targeting PLpro with drugs possessing

61 antiviral properties may have an additional benefit in inhibiting viral replication and

62 preventing the dysregulation of signaling cascades in infected cells that may lead to apoptosis

63 in surrounding uninfected cells [8].

65 vaccines manufactured by Pfizer-BioNTech, and Moderna's are authorized and recommended 
66 for the treatment of COVID-19. In addition, two vaccines manufactured by AstraZeneca and

67 Janssen are under Phase 3 clinical trials. However, with the identification of a new strain of

68 SARS-CoV-2, more efforts are needed to quickly identify novel drugs/vaccines. A traditional 69 approach to identify and develop a new antiviral agent against COVID-19 requires

70 comprehensive knowledge of genomics, potential protein targets, and pathogenesis of SARS-

71 CoV-2. Although the drugs developed using this approach would have a better anti-

72 coronavirus effect, it would take around 10-12 years to develop drugs with proper approval to

73 use clinically [9]. Another approach to identifying potent inhibitors of SARS-CoV-2 is the

74 repurposing of known drugs. This approach's main advantage is that the dosage, efficacy, and

75 potential side effects of drugs will be available beforehand, as they have been already

76 approved to treat various infections. However, the drug-repurposing approach's disadvantages

77 are its broad-spectrum nature (thus cannot be used against SARS-CoV-2 infection in a

78 targeted manner) and the associated side effects. Nevertheless, an in silico approach to

79 identify novel inhibitors of SARS-CoV-2 is to screen different databases for a molecule that

80 may have therapeutic potential against COVID-19 [8]. Computational approaches including

81 homology modeling, virtual screening by docking and molecular dynamics is a fast and time

82 saving approach to identify lead compounds against SARS-CoV-2 drug targets [10-13].

83 Natural compounds and herbal medicines offer a rich resource for a novel treatment

84 for the SARS-CoV-2 infection. In China, plant-based medicines to fight infectious diseases

85 employing Chinese traditional medicines have raised hope in the fight against the global

86 pandemic situation (WHO). Previously, plant-derived natural products with diverse biological

87 properties have been shown to possess inhibitory potential against Mpro and PLpro [14,15]. 
88 In this study, we have screened a natural compounds database and identified

89 Proanthocyanidin and Rhapontin as potential inhibitors of SARS-CoV-2 Mpro and PLpro

90 using computational approaches such as high throughput virtual screening, molecular docking

91 (standard precision and extra precision), free energy calculations, and molecular dynamics

92 simulation. The results of this study may provide crucial information to the scientific

93 community to develop Proanthocyanidin and Rhapontin into potential therapeutic intervention

94 against COVID-19 after validating their antiviral effects in vitro and in vivo.

\section{Materials and Methods}

\section{Pre-processing of ligands}

97 A library of natural products (L1400) containing 2267 unique and diverse ligand molecules

98

99

100

101

102

103

104

105

106

107

108

109 was retrieved from Selleck Inc (www.selleck.com). As described previously, the ligands were preprocessed using the "LigPrep tool (Schrodinger-2018-4, LLC, NY, USA)" by generating different ionization states at pH 7.0 \pm 2.0 employing "Epik tool (Schrodinger-2018-4, LLC, USA, NY) and the removal of any salt [16]. A maximum of 32 different conformational states of a given ligand was produced, and their energies were minimized using OPLS3e forcefield.

\section{Preprocessing of target proteins}

The crystal structures of SARS-CoV-2 proteases Mpro (PDB Id: 6LU7) and PLpro (PDB ID: 6W9C) were downloaded from the RCSB protein data bank (www.rcsb.org/pdb). The crystal structure of Mpro (Resolution: $2.16 \AA$ ) has been reported with a bound inhibitor N3, while that of PLpro (Resolution: $2.70 \AA$ ) has been reported without any inhibitor. Prior to virtual screening and molecular docking, the proteins were preprocessed using "Protein preparation wizard (Schrodinger-2018-4, LLC, NY, USA)" as reported earlier [17,18]. Briefly, missing 
hydrogen atoms were added, and then appropriate bond orders were allotted. The

111 crystallographic water molecules establishing less than three contacts with protein were

112 removed. Further, any missing side chains or loops modelled with the "Prime module

113 (Schrodinger-2018-4, LLC, NY, USA)". Finally, a hydrogen-bond network was generated on

114 the processed proteins and their energies were minimized employing using OPLS3e

115 forcefield. Grid box around the substrate binding sites of both the proteins were generated by

116 means of "Receptor-grid generation tool (Schrodinger-2018-4, LLC, NY, USA)". The

117 dimension of grid box used was $88 \times 88 \times 88 \AA$, and $78 \times 78 \times 78 \AA$ for Mpro, and PLpro

118 respectively. Prior to docking, the structure of target proteins was energy minimized using

119 OPLS3e forcefield.

120 High throughput virtual screening, standard-and extra-precision molecular docking

121 The natural product library was first screened against the target proteins (Mpro and

122 PLpro) by high throughput virtual screening (HTVS) using "Glide (Schrodinger-2018-4,

123 LLC, NY, USA)" as reported formerly [19]. The top-scoring 50 ligands against each target

124 proteins (docking energy range: -5.424 to $-11.212 \mathrm{kcal} / \mathrm{mol}$ for $\mathrm{Mpro}$; -5.185 to -6.973

$125 \mathrm{kcal} / \mathrm{mol}$ for PLpro) were selected and subjected to standard-precision (SP) molecular docking

126 with default settings. Finally, the best 6 ligands with the lowest binding energies against each

127 protein were again subjected to molecular docking using extra-precision (XP) mode. The

128 scaling factor and partial charge cut-off in XP docking were set to 0.80 and 0.15 , respectively.

129 The results were analyzed and visualized in "Maestro (Schrodinger-2018-4, LLC, NY, 130 USA)". The binding affinity $\left(K_{\mathrm{d}}\right)$ of the ligand was calculated from the docking free energy $131(\Delta G)$ with the help of equation given below [20,21]. 


$$
\Delta G=-R T \ln K_{\mathrm{d}}
$$

133 where, $R$ and $T$ were Boltzmann gas constant $(=1.987 \mathrm{cal} / \mathrm{mol} / \mathrm{K})$ and temperature $(=298 \mathrm{~K})$

134 respectively.

135 Further, the ligand efficiency (LE) which measures the validation of docking interactions was 136 estimated using the relation:

$$
\mathrm{LE}=\Delta G / \mathrm{N}
$$

138 where, $\Delta G$ is the docking free energy, and $\mathrm{N}$ is the number of non-hydrogen atoms or heavy 139 atoms in the ligand.

Validation of docking protocol by enrichment calculation

The validity of the docking procedure adopted in this study was conducted by using an enrichment calculator in "Maestro (Schrodinger-2018, LLC, NY, USA)" as described earlier

143 [22]. It evaluates the docking procedure's ability to identify active ligands from a pool of

144 inactive decoys and place them in the top percentage of a ranked database. Standard inactive

145 ligands or decoy set of Schrodinger was employed for the enrichment purpose. The area under

146 the curve (AUC) and enrichment factor was calculated at 1 and 20\%, respectively [23].

\section{Calculations of binding free energy using Prime/MM-GBSA}

The binding free energy of protein-ligand interaction was calculated using the

$$
\Delta G=E(\text { complex })_{\text {minimized }}-\left[E(\text { ligand })_{\text {minimized }}+E(\text { protein })_{\text {minimized }}\right]
$$


155 To calculate MM-GBSA, ligand atoms were set unrestricted while the atoms of protein were

156 fixed, and based on free energies, generated protein-ligand poses were ranked.

\section{Molecular dynamics (MD) simulation}

The dynamics and stability of the protein-ligand complex were measured by

159 performing molecular dynamics simulation using "Desmond (Schrodinger-2018-4, LLC, NY,

160 USA), reported earlier [25]. Succinctly, the protein-ligand complex was positioned in an

161 orthorhombic simulation box, keeping a minimum distance of $10 \AA$ from the box's

162 boundaries. The TIP3P explicit water model was used to solvate the simulation box, and the

163 system was neutralized by adding proper counter ions. The concentration of $\mathrm{NaCl}$ was

164 adjusted to $150 \mathrm{mM}$ to simulate the biological condition. The complete system was pre-

165 equilibrated for 2000 iterations with a convergence criterion of $1 \mathrm{kcal} / \mathrm{mol} / \AA$ using OPLS3e

166 force field. Lastly, simulation (50 ns) was executed employing NTP ensemble at $300 \mathrm{~K}$ and 1

167 bar, using OPLS3e forcefield. Nose-Hoover Chain thermostat and Matrtyna-Tobias-Klein

168 barostate were employed to maintain the temperature and pressure constant [26,27]. During

169 the simulation process, a time step of $2 \mathrm{fs}$ was set, and at every $10 \mathrm{ps}$, the energies and

170 structures were recorded in the trajectory.

171 Physiological and ADMET properties

172 The physicochemical and ADMET (Absorption, Distribution, Metabolism, Excretion,

173 and Toxicity) properties of the most potent ligands were assessed employing "QikProp

174 (Schrodinger-2018-4, LLC, NY, USA)” as reported earlier [28]. QikProp predicts ADMET

175 properties on the basis of complete 3D molecular structure of a ligand. The following

176 descriptors were evaluated: molecular weight (Mol_MW), solvent accessible surface area 
177 (SASA), hydrophobic component of SASA (FOSA), hydrophilic component of SASA

178 (FISA), polar component of SASA (PISA), weakly polar component of SASA (WPSA),

179 hydrogen bond donor (donorHB), hydrogen bond acceptor (accptHB), number of rotatable

180 bonds, polarizability (QPpolrz), hexadecane/gas partition coefficient (QPlogPC16),

181 octanol/gas partition coefficient (QPlogPoct), water/gas partition coefficient (QPlogPw),

182 octanol/water partition coefficient (QPlogPo/w), solubility (QPlogS), conformation-dependent

183 solubility (CIQPlogS), blockage of HERG K ${ }^{+}$channel (QPlogHERG), CACO permeability

184 (QPPCaco), blood/brain partition coefficient (QPlogBB), MDCK cell permeability

185 (QPPMDCK), skin permeability (QPlogKp), binding to human serum albumin (QPlogKhsa)

186 and \% oral absorption.

187 Results and Discussion

The ongoing global COVID-19 pandemic has revealed the reality of our preparedness

in fighting human coronaviruses. The outbreaks of SARS-CoV in 2003 and MERS in 2012

190 initiated extensive research in developing drugs against zoonotic coronaviruses, but still, no

191 drugs are available in the market. One of the reasons might be the viral genome's evolving

192 nature, which limited the progression of initial findings to the clinical stages [29]. The fastest

193 way to tackle this situation is to repurpose drugs available to treat other diseases since their

194 dosages, side effects, and ADMET properties are well known. However, drug-repurposing's

195 main drawback is the drug's inability to treat COVID-19 in a targeted manner. Moreover, the

196 repurposed drug may behave in an unpredicted manner in the altered pathophysiological

197 condition of COVID-19 patients. Thus, identifying novel inhibitors of SARS-CoV-2 from

198 natural sources is a viable alternative as they are believed to be less toxic [30,31]. 
199

200

201

202

203

204

205

206

207

208

209

210

211

212

213

214

215

216

217

218

219

220

\section{Virtual screening of natural compounds against Mpro and PLpro}

Kuntz et al. (1982) first described molecular docking in 1982, and since then, it has

become the principal idea in virtual screening of structure-based drug design [32]. In this

study, the high throughput virtual screening of natural compounds led us identifying 50

potential drug candidates against Mpro and PLpro (Supplementary Table S1 and S2).

Standard precision (SP) and extra precision (XP) molecular docking further shortlisted the

potential compounds to 6 each against Mpro and PLpro (Table 1). The most promising

compounds against Mpro were Polymyxin B, Complanatuside, Kaempferol-3-O-

glucosrhamanoside, Proanthocyanidin, Typhaneoside, and Amikacin. Likewise, the most

potential compounds against PLpro were Secoisolariciresinol diglucoside, Sophoricoside,

Eriocitrin, (+)-Catechin, Rhapontin, and Nadide. The XP molecular docking scores of

Polymyxin B, Complanatuside, Kaempferol-3-O-glucosrhamanoside, Proanthocyanidin,

Typhaneoside, and Amikacin towards Mpro were -10.704, -10.599, -10.566, -10.515, -9.442,

and $-9.103 \mathrm{kcal} / \mathrm{mol}$, respectively (Table 1 ). Similarly, the XP molecular docking score of

Rhapontin, Secoisolariciresinol diglucoside, Eriocitrin, Sophoricoside, Nadide, and (+)-

Catechin was $-10.022,-9.374,-8.677,-8.066,-5.629$, and $-5.006 \mathrm{kcal} / \mathrm{mol}$ against PLpro

(Table 2). Further, the free energy of interaction between proteins and natural compounds

were determined by performing Prime/MM-GBSA on all the selected compounds listed in

Table 1. The Prime/MM-GBSA energy of Proanthocyanidin and Rhapontin were the lowest, -

89.98 and $-61.39 \mathrm{kcal} / \mathrm{mol}$ against Mpro and PLpro, respectively (Table 1). 
221 Validation of receiver operating characteristic (ROC) curve

The validity of docking procedures was established by performing enrichment

223 calculation on the shortlisted 6 compounds with the lowest docking energies against each

224 target proteins. The enrichment analysis was performed by plotting ROC curves and checking

225 the adopted docking protocol's ability to distinguish the active compounds from a pool of

226 decoy set of inactive compounds (Figure 1). The ROC values of XP docking against Mpro

227 and PLpro were 0.99 and 0.97, respectively. The BEDROC scores for Mpro and PLpro

228 docking were 0.929 at $\alpha-0.0556$ and 0.788 at $\alpha-0.0714$, respectively. Moreover, the area under

229 curve (AUC) values of compounds docked to Mpro and PLpro were 0.99 and 0.96,

230 respectively. Since the AUC values for both Mpro and PLpro docking were significantly

231 greater than 0.5 , it is clear that the docking procedure adopted in this study is accurate in

232 predicting the active compounds from an extensive database. Moreover, root mean square

233 deviation (RMSD) between the crystal structure pose and docked pose of Mpro bound N3 was

234 elucidated and was estimated to be $1.1564 \AA$ (Supplementary Figure S1).

235 Interaction between proteins and inhibitors

236 In recent studies, Mpro and PLpro of SARS-CoV-2 have been illustrated as the best

237 drug targets due to their significant participation in transcription and replication of virus genes

238 and modulating the host's innate immune system [33]. Mpro and PLpro have been reported to

239 be indispensable for processing the polyprotein chain of SARS-CoV-2, which is translated

240 from viral RNA [34]. Moreover, the availability of detailed protein structures, knowledge of

241 enzymes' drug-binding pockets, and conserved nature of these proteins amongst

242 coronaviruses, make them ideal target for drug development purposes. 
245 inhibitor to decipher a strategy that may be exploited in a rapid drug discovery endeavor [35].

246 The 3D structure of Mpro is composed of 306 amino acid residues with three different

247 domains (I-III). The amino acid residues 8-101 encompass domain I, while amino acid

248 residues 102-184 are present in domain II acquiring antiparallel beta structure. Domain III

249 spanning amino acid residues 201-303 comprises five $\alpha$-helices organized mainly into an

250 antiparallel globular cluster, connected to domain II by an extended loop region (amino acid

251 residues 185-200). The substrate-binding site of Mpro is positioned in between domain 1 and

252 II in a deep pocket, lined with a Cys41-His145 catalytic dyad, as previously reported in Mpro

253 of other coronaviruses [36].

254 A detailed analysis of Mpro and Proanthocyanidin's interaction implicates that the

255 inhibitor was bound to the substrate-binding site of Mpro. The Mpro-Proanthocyanidin

256 complex was stabilized by six hydrogen bonds with Asn142, Cys 145, His163, Glu166,

257 Gln189, and Thr190 (Figure 2A and Table 2). The amino acid residues responsible for van der 258 Waals' interaction with Proanthocyanidin were Phe140, Leu141, Gly143, Ser144, His164, 259 Met165, Leu167, Pro168, Hie172, Val186, Asp187, Arg188, Ala191, and Gln192. Further, 260 there was a hydrophobic interaction between the active site residue Hie41 and

261 Proanthocyanidin. An analysis of Mpro from SARS-CoV (PDB ID: 1UK3) and SARS-CoV-2

262 (PDB ID: 6LU7) suggests that only 12 out of 306 amino acid residues i.e. $3.9 \%$ differed

263 between SARS-CoV and SARS-CoV-2 (Supplementary Figure S2). Most of the mutations in

264 SARS-CoV-2 are synonymous in nature, and therefore did not affect the overall structure and 
265 function of Mpro. Interestingly, the amino acid residues such as Hie41, Asn142, Cys145,

266 His163, Glu166, Gln189 and Thr190 of Mpro forming hydrogen bonds and hydrophobic

267 interactions were highly conserved in both SARS-CoV and SARS-CoV-2 (Supplementary

268 Figure S3A). The docking energy of interaction between Mpro and Proanthocyanidin was

269 assessed to be $-10.566 \mathrm{kcal} / \mathrm{mol}$, which corresponded to a binding affinity of $5.62 \times 10^{7} \mathrm{M}^{-1}$

270 (Table 2). Also, the ligand efficiency of Proanthocyanidin to bind Mpro was estimated to be -

$2710.153 \mathrm{kcal} / \mathrm{mol}$ (Table 2). Recently, Kumar and Roy (2020) have developed a QSAR model

272 against SARS-CoV-2 Mpro to identify potent inhibitors by screening large databases [37].

273 They identified several features which may enhance the potency of Mpro inhibitors such as

274 the frequency of O-S bond at topological distance 6, number of thiophene rings, compound's

275 shape and the length of acyclic chain. Conversely, features such as pyridine rings, presence of

$276 \mathrm{CH}_{2} \mathrm{X}_{2}$ fragment, number of imides, and the presence of $\mathrm{N}-\mathrm{O}$ fragment at topological distance

2772 were detrimental to Mpro inhibition potential. An analysis of Proanthocyanidin confirmed

278 that it lacks all the features of QSAR model which were detrimental to the inhibition of Mpro.

279 Proanthocyanidins are condensed tannins possessing a plethora of pharmacological and

280 biological properties [38]. In term of chemical composition, Proanthocyanidins are basically

281 oligomeric flavonoids such as oligomers of catechin and epicatechin and their gallic acid

282 esters. They are naturally present in flowers, nuts, fruits, barks, and numerous plants like

283 apples, pine, cinnamon, aronia, cocoa, and grapes. Proanthocyanidins have been reported to

284 possess antiviral, anti-microbial, anticancer, antioxidant, anti-diabetes, immunomodulatory

285 activities in vitro [39]. Most notably, the antiviral activity of proanthocyanidins has been

286 reported against herpes simplex virus-1 (HSV-1) [40], porcine reproductive and respiratory 
287

288

289

290

291

292

293

294

295

296

297

298

299

300

301

302

303

304

305

306

307

308

syndrome virus (PRRSV) [41], coxsackie B virus (CBV) [42], canine distemper virus (CDV) [42]. Moreover, Procyanidins have been reported to display moderate inhibition of SARS-

CoV infection [43].

Investigation of PLpro-Rhapontin interaction

The 3D structure of SARS-CoV-2 PLpro resembles a thumb-palm-finger architecture of a right-hand, wherein the active site is located at the interface of palm and thumb subdomains and characterized by the presence of a catalytic triad Cys111-His272-Asp286.

During catalysis, Trp106 also plays a significant role in stabilizing the transition-state intermediates $[44,45]$. PLpro modulates the host's innate immune response against the virus through its de-ubiquitin and de-ISGylating activities [46]. The N-terminal end of PLpro has a ubiquitin-like domain (UBL), which recognizes ubiquitin (Ub) and UBL protein ISG15 (Interferon-induced gene 15) tagged proteins with LXGG recognition sequence at their Cterminal end $[45,47,48]$.

A close investigation of the PLpro-Rhapontin complex suggests that Rhapontin was bound to the substrate-binding site of PLpro, situated at the cleft of thumb and palm domains. Rhapontin formed four hydrogen bonds with Trp106, Gly271, Hie272, and Asp286; and hydrophobic interaction with the active site residue Hie272 (Figure 2B and Table 2). An analysis of PLpro from SARS-CoV (PDB ID: 2FE8) and SARS-CoV-2 (PDB ID: 6W9C) suggests that only 54 out of 316 amino acid residues i.e. $17.2 \%$ differed between SARS-CoV and SARS-CoV-2 (Supplementary Figure S4). Most of the mutations in SARS-CoV-2 are synonymous in nature, and therefore did not affect the overall structure and function of PLpro. It is worth to note that, the amino acid residues such as Trp106, Gly271, Hie272 and 
Asp286 of PLpro forming hydrogen bonds and hydrophobic interactions were highly conserved in both SARS-CoV and SARS-CoV-2 (Supplementary Figure S3B). Further, the amino acids responsible for van der Waals' interaction with Rhapontin were Lys105, Asn109, Cys111, Leu162, Gln269, Cys270, Tyr273, Gly287, Ala288, and Leu289. The respective docking energy and docking affinity of PLpro towards the Rhapontin complex were estimated as $-10.022 \mathrm{kcal} / \mathrm{mol}$ and $2.24 \times 10^{7} \mathrm{M}^{-1}$ (Table 2). Moreover, the ligand efficiency of Rhapontin to bind PLpro was predicted to be $-0.186 \mathrm{kcal} / \mathrm{mol}$ (Table 2). Previously, a QSAR model of PLpro inhibitor has been reported by Amin et al (2020), wherein they showed that an active inhibitor of PLpro contains an aromatic ring and electronegative atoms [49].

Rhapontin fulfilled the criteria of QSAR model and emerged as a potent inhibitor of PLpro. Rhapontin or rhaponticin is a stilbene-type glycoside abundantly found in medicinal plants of the Rheum genus (Polygonaceae). Rhapontigenin, the aglycone form of rhapontin, is the biologically active form displaying various activities such as antioxidant, anti-fungal, antithrombotic, anti-inflammatory effect and anti-proliferative activity [50].

\section{Analysis of molecular dynamics (MD) simulation}

MD simulation is a primary tool in computational chemistry to get comprehensive information on the fluctuations and conformational changes of proteins and ligands in a timedependent manner [51]. To investigate the structure and dynamics of proteins (Mpro and PLpro) complex with their respective inhibitors (Proanthocyanidin and Rhapontin), we have performed MD simulation for $50 \mathrm{~ns}$, and the results are presented below. 
331

332

333

334

335

336

337 338

339

340

341

342

343

344

345

346

347 348

Root mean square deviation (RMSD)

RMSD is a fundamental property to investigate the changes in the conformation and compactness of a protein occurred due to the binding of a ligand [52]. An analysis of the deviation in $\mathrm{C} \alpha$-atoms of Mpro with respect to the initial frame of protein alone (blue line) or in a complex (brown line) with Proanthocyanidin revealed a significant fluctuation in RMSD values for the initial $20 \mathrm{~ns}$ of simulation time (Figure 3). However, for the later part of the simulation (21-50 ns), the RMSD values were within the maximum allowed value of $2.0 \AA$, thus indicating a stable Mpro-Proanthocyanidin complex formation (Figure 3A). Similarly, the variation in $\mathrm{C} \alpha$-atoms of PLpro alone (blue line) with respect to its initial frame suggests that the protein attained a stable conformation at the start of the simulation. The RMSD values of PLpro alone remain within 1.2-2.4 $\AA$ throughout the simulation. Although the RMSD values in $\mathrm{C} \alpha$-atoms of PLpro-Rhapontin complex (brown line) with respect to the initial frame fluctuated enormously for $0-25 \mathrm{~ns}$, it was stabilized within the acceptable limit of $2.0 \AA$ for the remaining simulation time (Figure 3B). At the start of the simulation, the minor fluctuations in RMSD values were expected due to the entry of big ligands such as Proanthocyanidin and Rhapontin into the substrate binding cavity of Mpro and PLpro respectively. However, on the formation of stable interactions, the RMSD values of Mpro as well as PLpro get stabilized.

Root mean square fluctuation (RMSF)

RMSF values of Mpro and PLpro were calculated to gain an insight into the fluctuation of side chains and hence local conformation of a protein during the simulation (Figure 3C,D). The variation in RMSF of proteins (teal line) along their chain length was 
353 compared to their respective B-factors (brown line), which were determined experimentally

354 during X-ray crystallography. Moreover, the vertical bars in light pink, teal and white color

355 represent regions of the protein participating in the formation of $\alpha$-helices, $\beta$-sheets and loops

356 respectively. The vertical green lines on the $\mathrm{X}$-axis show the position of amino acid residue

357 forming an interaction with the protein.

358 As evident from Figure 3C, the N- and C-terminals of Mpro tend to fluctuate more as

359 they remain free from the rest of the protein. Overall, Mpro remained stable upon interacting

360 with Proanthocyanidin as there were only minor fluctuations in the loop regions (white bars)

361 of the protein. Similarly, the overall structure of PLpro in the presence of Rhapontin also

362 remains consistent throughout the simulation, as evident from the minor fluctuations in the $\alpha$ -

363 helices and $\beta$-sheets of PLpro. However, some significant fluctuations in PLpro were

364 observed in the loop regions of protein due to the binding of a large molecule like Rhapontin

365 (Figure 3D).

366 Protein-ligand interaction analysis

367 An analysis of molecular interaction between Mpro and Proanthocyanidin during MD

368 simulation revealed that the Mpro-Proanthocyanidin complex was mainly stabilized by

369 hydrogen bonding, a minor contribution of hydrophobic interactions and water bridges

370 (Figure 4A). The catalytic residue His41 of Mpro interacted with Proanthocyanidin for about

$37150 \%$ of simulation through hydrogen bonding, hydrophobic interaction, and water bridges.

372 Similarly, another catalytic residue of Mpro i.e., Cys 145 formed a hydrogen bond and water

373 bridges with Proanthocyanidin for $100 \%$ and $40 \%$ simulation time, respectively. Some other

374 residues, such as Glu166, Thr190, and Gln192, formed a hydrogen bond with 
375 Proanthocyanidin for $90-100 \%$ simulation. The residues Pro168 and Gln 189 were engaged in

376 hydrophobic and hydrogen bonding for 50 and $40 \%$ simulation time (Figure 4A). The total

377 number of contacts between Mpro and Proanthocyanidin throughout the simulation was

378 estimated in the range of 4-16, with an average of 9 contacts (Figure 4B, upper panel). The

379 behavior of amino acid residues in making interaction with Proanthocyanidin as a function of

380 simulation is also depicted (Figure 4B, lower panel). It appears that His41, Cys145, Glu166,

381 Pro168, Gln189, Thr190, and Gln192 participated in the interaction with Proanthocyanidin for

382 most of the simulation time.

383 An insight into the MD simulation of PLpro-Rhapontin complex suggested that it was

384 stabilized by hydrogen bonding, hydrophobic interactions, and a small contribution of water

385 bridges (Figure 4C). The catalytic residue Trp106 interacted with Rhapontin for about 95\% of

386 simulation time through hydrogen bonding, hydrophobic interaction, and water bridges. The

387 residue Asp108 and Asn109 also contributed significantly in stabilizing the PLpro-Rhapontin

388 complex by forming a hydrogen bond for $30 \%$ and $55 \%$ simulation time respectively. Other

389 residues such as Glu161, Cys270, Gly271, and Asp286 also formed hydrogen bonds and

390 water bridges with PLpro for more than 30\% simulation time (Figure 4C). The total number

391 of contacts between Mpro and Proanthocyanidin throughout the simulation was estimated in

392 the range of 1-14, with an average of 8 contacts (Figure 4D, upper panel). The behavior of

393 amino acid residues in making interaction with Rhapontin as a function of simulation is also

394 depicted (Figure 4D, lower panel). It was found that Trp106, Asp108, and Asn109

395 participated in the interaction with Rhapontin for most of the simulation time. 
401

402

403

404

405

406

407

408

409

410

411

412

Secondary structure analysis

The binding of a ligand to a protein often leads to significant changes in protein's secondary structure. Here, the changes in the secondary structure elements (SSE) in Mpro and PLpro upon binding their respective inhibitors i.e., Proanthocyanidin and Rhapontin, were monitored throughout the simulation (Figure 5). During MD simulation, the total SSE of Mpro bound with Proanthocyanidin was estimated to be $46 \%(\alpha$-helix $=21 \%$ and $\beta$-sheets $=$ $25 \%$ ), which was similar to the X-ray determined SSEs 52\% comprising $27 \% \alpha$-helix and $25 \% \beta$-sheets (Figure 5A, upper panel). Similarly, the SSEs of PLpro bound with Rhapontin was determined to be $51 \%(\alpha$-helix $=24 \%$ and $\beta$-sheets $=27 \%)$ during MD simulation, which was similar to the X-ray determined SSEs $59 \%$ comprising $28 \% \alpha$-helix and $31 \% \beta$-sheets (Figure 5B, lower panel). Moreover, the contribution of individual amino acid residues in the formation of SSEs as a function of simulation time is also represented for Mpro (Figure 5A, lower panel) and PLpro (Figure 5B, lower panel). The results clearly show that the binding of inhibitors (Proanthocyanidin and Rhapontin) to proteins (Mpro and PLpro) did not significantly alter their SSEs.

Analysis of radius of gyration (rGyr) and solvent accessible surface area (SASA)

The radius of gyration $(\mathrm{Rg})$ is a critical factor to access the folding state and overall conformation of a protein under different conditions [53]. The rGyr values of

Proanthocyanidin and Rhapontin were measures to assess the compactness of ligand bound to their respective proteins Mpro and PLpro as a function of simulation time (Figure 6A). The rGyr of Mpro showed a consistent behavior throughout the simulation, while there was a small fluctuation in the rGyr of PLpro for the initial $10 \mathrm{~ns}$. In the later part of the simulation 
419 (11-50 ns), the ligands' rGyr was stabilized within acceptable limits. The average values of

420 rGyr for Proanthocyanidin and Rhapontin were calculated as 4.42 and $5.50 \AA$, respectively.

421 SASA is measured as the volume of protein exposed to the surrounding solvent.

422 SASA of a protein behaves differently in different conditions and thus gives an insight into its

423 conformational behavior [54]. The dependence of SASA as a function of the simulation was

424 evaluated to measure the exposure of Proanthocyanidin and Rhapontin, bound to Mpro and

425 PLpro, respectively, to the solvent (Figure 6B). SASA of Proanthocyanidin and Rhapontin

426 showed a consistent behavior with fluctuations remained within acceptable limits throughout

427 the simulation time. The average values of SASA of Proanthocyanidin and Rhapontin were

428 estimated as 276.4 and $282.1 \AA$, respectively. The rGyr and SASA results confirm the stable

429 nature of ligands Proanthocyanidin and Rhapontin bound at the substrate site of their

430 respective proteins Mpro and PLpro.

431 Investigation of physicochemical and ADMET properties

432 In silico methods have been established for the prediction of drug-likeness properties

433 of any compound under computer-aided discovery. A high-quality drug candidate should

434 possess appropriate efficacy against target proteins and display good ADMET properties [55].

435 The physicochemical and ADMET properties of Proanthocyanidin and Rhapontin were

436 determined employing "QikProp (Schrodinger-2018, LLC, NY, USA)” and listed in Table 3.

437 The molecular weight of Proanthocyanidin and Rhapontin were within the acceptable range of

$438 \quad 150-750 \mathrm{~g} / \mathrm{mol}$ of a drug-like molecule. Other physicochemical properties such as the number

439 of volume, rotatable bonds, hydrogen bond donor, and hydrogen bond acceptor of

440 Proanthocyanidin and Rhapontin were higher than the acceptable range of an efficient drug- 
441 like molecule. The polarizability (QPpolrz), which defines the drug-like properties of any

442 compound, was found to be 54.708 for Proanthocyanidin and 38.114 for Rhapontin (Table 3).

443 The ADMET property, such as solvent accessible surface area (SASA) of

444 Proanthocyanidin and Rhapontin, were within the acceptable range of 300-1000. In addition

445 to SASA, the hydrophobic (FOSA) and hydrophilic (FISA), polar (PISA), and weakly polar

446 (WPSA) components of SASA were within limits for Rhapontin. Similarly, Proanthocyanidin

447 obeys the laws of a drug-like substance in terms of FOSA, PISA, and WPSA. The value of

448 FISA for Proanthocyanidin was a little higher than the prescribed limit (Table 3). The

449 hexadecane/gas (QPlogPC16), octanol/gas (QPlogPoct), water/gas (QPlogPw), octanol/water

$450(\mathrm{QP} \operatorname{logPo} / \mathrm{w})$ partition coefficients, and solubility (QPlogS), conformation-dependent

451 solubility (CIQPlogS), CACO permeability (QPPCaco), blood/brain partition coefficient

452 (QPlogBB), and skin permeability (QPlogKp) of Rhapontin were within or close to the

453 acceptable limits of a drug-like molecule. The values of blockage of HERG K+ channels

454 (QPlogHERG), MDCK cell permeability (QPPMDCK), and binding to human serum albumin

455 (QPlogKhsa) of Rhapontin were out of the range prescribed for a drug-like molecule.

456 Similarly, QPlogPC16 and QPlogPoct of Proanthocyanidin were marginally higher

457 than the acceptable limit. The $\mathrm{QP} \log \mathrm{Pw}, \mathrm{QP} \log \mathrm{Po} / \mathrm{w}$ partition coefficients, and solubility

458 (QPlogS) of Proanthocyanidin were within the range of a drug-like molecule (Table 3). The

459 values of CIQPlogS, QPlogHERG, QPPCaco, QPlogBB, and QPPMDCK for

460 Proanthocyanidin were not fit into the acceptable range. On the other hand, the values of

461 QPlogKp and QPlogKhsa of Proanthocyanidin were within the prescribed limit of a drug-like 
462 molecule. Further, the human oral absorption (\%) of Proanthocyanidin was 0, while

463 Rhapontin was 40, a value characteristic of a drug-like molecule (Table 3).

464 Conclusion

465 In the present study, a natural compound library (L1400, available at Selleck Inc.) has

466 been screened virtually, targeting Mpro and PLpro proteases of SARS-CoV-2. On the basis of

467 molecular docking and free energy calculations, Proanthocyanidin and Rhapontin are

468 identified as the most effective inhibitors of Mpro and PLpro, respectively. The stability and

469 dynamics of Mpro-Proanthocyanidin and PLpro-Rhapontin complexes have been established

470 by performing molecular dynamics simulation. Moreover, the physicochemical and ADMET

471 properties of Proanthocyanidin and Rhapontin revealed that Rhapontin has almost all the

472 properties of a drug-like molecule, while Proanthocyanidin could serve as a scaffold for the

473 development of a potent drug molecule. The compounds identified here could potentially

474 inhibit viral proteases, however, they are not approved drugs for treating any disease. The

475 limitation of this study is that it is a purely computational study and does not include any data

476 on the functional validation of docking results in vitro or in vivo. Thus, the results of this

477 study require in vitro, ex vivo and in vivo validation for the tested natural compounds

478 (Proanthocyanidin and Rhapontin) before clinical usage.

479 Supplementary Information: The following supplementary information is available at the

480 online version of this article. Table S1: Standard precision (SP) molecular docking of SARS-

481 CoV-2 Mpro with top 50 natural compounds from L1400 library of Selleck Inc.; Table S2:

482 Standard precision (SP) molecular docking of SARS-CoV-2 PLpro with top 50 natural

483 compounds from L1400 library of Selleck Inc. Figure S1: Root mean square deviation 
(RMSD) between the docked pose and crystal pose of N3 ligand bound to Mpro. Figure S2:

Pairwise sequence alignment of Mpro derived from SARS-CoV and SARS-CoV-2. The

residues inside box are involved in hydrogen bonds and hydrophobic interactions. Figure S3:

Superimposition of (A) SARS-CoV-2 Mpro-Proanthocyanidin, and (B) SARS-CoV-2 PLpro-

Rhapontin active sites with that of Mpro and PLpro from SARS-CoV. Amino acid residues

depicted in purple and golden colors belong to Mpro of SARS-CoV and SARS-CoV-2,

respectively. Similarly, amino acid residues depicted in magenta and blue colors belong to

PLpro of SARS-CoV and SARS-CoV-2, respectively. Amino acid residues depicted in purple

alignment of PLpro derived from SARS-CoV and SARS-CoV-2. The residues inside box are

involved in hydrogen bonds and hydrophobic interactions.

\section{References}

1. Schoeman D, Fielding BC. Coronavirus envelope protein: current knowledge. Virol J 2019; 16: 69 .

2. She J, Jiang J, Ye L, Hu L, Bai C, Song Y. 2019 novel coronavirus of pneumonia in Wuhan, China: emerging attack and management strategies. Clin Transl Med 2020; 9: 19.

3. Woo PCY, Huang Y, Lau SKP, Yuen KY. Coronavirus genomics and bioinformatics analysis. Viruses 2010; 2: 1804-1820.

4. Song Z, Xu Y, Bao L, Zhang L, Yu P, Qu Y, et al. From SARS to MERS, Thrusting Coronaviruses into the Spotlight 2019; $11: 59$.

5. Romano M, Ruggiero A, Squeglia F, Maga G, Berisio R. A Structural View of SARSCoV-2 RNA Replication Machinery: RNA Synthesis, Proofreading and Final Capping. Cells 2020; 9: 1267.

6. Xue X, Yu H, Yang H, Xue F, Wu Z, Shen W, et al. Structures of Two Coronavirus Main Proteases: Implications for Substrate Binding and Antiviral Drug Design. J Virol 2008; 82:2515-2527.

7. Báez-Santos YM, St. John SE, Mesecar AD. The SARS-coronavirus papain-like protease: Structure, function and inhibition by designed antiviral compounds. Antivir Res 2015; 115: 21-38.

8. Xu J, Li X, Jiang B, Feng X, Wu J, Cai Y, et al. Antiviral Immunotoxin Against Bovine herpesvirus-1: Targeted Inhibition of Viral Replication and Apoptosis of Infected Cell. Front Microbiol 2018; 9: 653. 
9. Zhou Y, Hou Y, Shen J, Huang Y, Martin W, Cheng F. Network-based drug repurposing for novel coronavirus 2019-nCoV/SARS-CoV-2. Cell Discov 2020; 6: 14.

10. Ojha PK, Krishna JG, Roy K, Leszczynski J. Therapeutics for COVID-19: from computation to practices-where we are, where we are heading to. Mol Divers 2020; https://doi.org/10.1007/s11030-020-10134-X

11. Reiner Z, Hatamipour M, Banach M, Pirro M, Al-Rasadi K, Jamialahmadi T, Radenkovic D, montecucco F, Sahebkar A. Statins and the COVID-19 main protease: in silico evidence on direct interaction. Arch Med Sci 2020; 16(3): 490-496.

12. Borgio JF, Alsuwat HS, Al Otaibi WM, Ibrahim AM, Almandil NB, Al Asoom LI et al. State-of-the-art tools unveil potent drug targets amongst clinically approved drugs to inhibit helicase in SARS-CoV-2. Arch Med Sci 2020; 16(3): 508-518.

13. Azeez SA, Ghalib Alhashim Z, Al Otaibi WM, Alsuwat HS, Ibrahim AM, Almandil NB et al. State-of-the-art tools to identify druggable protein ligand of SARS-CoV-2. Arch Med Sci 2020; 16(3): 497-507.

14. Jo S, Kim S, Shin DH, Kim MS. Inhibition of SARS-CoV 3CL protease by flavonoids. J Enzyme Inhib Med Chem 2020; 35: 145-151.

15. Jo S, Kim H, Kim S, Shin DH, Kim MS. Characteristics of flavonoids as potent MERSCoV 3C-like protease inhibitors. Chem Biol Drug Des 2019; 94: 2023-2030.

16. AlAjmi MF, Azhar A, Owais M, Rashid S, Hasan S, Hussain A, et al. Antiviral potential of some novel structural analogs of standard drugs repurposed for the treatment of COVID-19. J Biomol Struct Dyn 2020: 1-13.

17. Parvez MK, Rehman MT, Alam P, Al-Dosari MS, Alqasoumi SI, Alajmi MF. Plantderived antiviral drugs as novel hepatitis B virus inhibitors: Cell culture and molecular docking study. Saudi Pharm J 2019; 27: 389-400.

18. AlAjmi MF, Rehman MT, Hussain A, Rather GM. Pharmacoinformatics approach for the identification of Polo-like kinase-1 inhibitors from natural sources as anticancer agents. Int J Biol Macromol 2018; 116: 173-81.

19. Rehman M, AlAjmi M, Hussain A, Rather G, Khan M. High-Throughput Virtual Screening, Molecular Dynamics Simulation, and Enzyme Kinetics Identified ZINC84525623 as a Potential Inhibitor of NDM-1. Int J Mol Sci 2019; 20: 819.

20. Rehman MT, Shamsi H, Khan AU. Insight into the Binding Mechanism of Imipenem to Human Serum Albumin by Spectroscopic and Computational Approaches. Mol Pharm 2014; 11: 1785-97.

21. Rehman MT, Ahmed S, Khan AU. Interaction of meropenem with ' $N$ ' and ' $B$ ' isoforms of human serum albumin: a spectroscopic and molecular docking study. J Biomol Struct Dyn 2016; 34: 1849-64.

22. Verma P, Tiwari M, Tiwari V. In silico high-throughput virtual screening and molecular dynamics simulation study to identify inhibitor for AdeABC efflux pump of Acinetobacter baumannii. J Biomol Struct Dyn 2018; 36: 1182-1194.

23. Florkowski CM. Sensitivity, specificity, receiver-operating characteristic (ROC) curves and likelihood ratios: communicating the performance of diagnostic tests. Clin Biochem Rev 2008; 29 Suppl 1: S83-87.

24. Rehman MT, AlAjmi MF, Hussain A. Natural Compounds as Inhibitors of SARS-CoV-2 Main Protease (3CLpro): A Molecular Docking and Simulation Approach to Combat 
COVID-19. ChemRxiv 2020. https://doi.org/10.26434/chemrxiv.12362333.v2. 25. Al-Shabib NA, Khan JM, Malik A, Rehman MT, AlAjmi MF, Husain FM, et al. Molecular interactions of food additive dye quinoline yellow (Qy) with alpha-lactalbumin: Spectroscopic and computational studies. J Mol Liq 2020; 311: 113215.

26. Martyna GJ, Tobias DJ, Klein ML. Constant pressure molecular dynamics algorithms. J Chem Phy 1994; 101: 4177-4189.

27. Brańka AC. Nosé-Hoover chain method for nonequilibrium molecular dynamics simulation. Phys Rev E 2000; 61: 4769-4773.

28. Mohammad T, Shamsi A, Anwar S, Umair M, Hussain A, Rehman MT, et al. Identification of high-affinity inhibitors of SARS-CoV-2 main protease: Towards the development of effective COVID-19 therapy. Virus Res 2020; 288: 198102.

29. Guo YR, Cao QD, Hong ZS, Tan YY, Chen SD, Jin HJ, et al. The origin, transmission and clinical therapies on coronavirus disease 2019 (COVID-19) outbreak- An update on the status. Mil Med Res 2020; 7: 11.

30. Cinatl J, Morgenstern B, Bauer G, Chandra P, Rabenau H, Doerr HW. Glycyrrhizin, an active component of liquorice roots, and replication of SARS-associated coronavirus. Lancet 2003; 361: 2045-46.

31. Wang Z, Chen X, Lu Y, Chen F, Zhang W. Clinical characteristics and therapeutic procedure for four cases with 2019 novel coronavirus pneumonia receiving combined Chinese and Western medicine treatment. Biosci Trends 2020; 14: 64-68.

32. Kuntz ID, Blaney JM, Oatley SJ, Langridge R, Ferrin TE. A geometric approach to macromolecule-ligand interactions. J Mol Biol 1982; 161: 269-288.

33. Zhang L, Lin D, Sun X, Curth U, Drosten C, Sauerhering L, et al. Crystal structure of SARS-CoV-2 main protease provides a basis for design of improved a-ketoamide inhibitors. Science 2020; 368: 409-412.

34. Morse JS, Lalonde T, Xu S, Liu WR. Learning from the Past: Possible Urgent Prevention and Treatment Options for Severe Acute Respiratory Infections Caused by 2019-nCoV. ChemBioChem 2020; 21: 730-738.

35. Jin Z, Du X, Xu Y, Deng Y, Liu M, Zhao Y, et al. Structure of Mpro from COVID-19 virus and discovery of its inhibitors. Nature 2020; 582: 289-293.

36. Palese LL. The Structural Landscape of SARS-CoV-2 Main Protease: Hints for Inhibitor Search. ChemRxiv 2020. https://doi.org/10.26434/chemrxiv.12209744.v1.

37. Kumar V, Rou K. Development of a simple, interpretable and easily transferable QSAR model for quick screening antiviral databases in search of novel 3Clike protease (3CLpro) enzyme inhibitors against SARS-CoV diseases. SAR QSAR Environ Res 2020; 31: 511526

38. de la Iglesia R, Milagro FI, Campión J, Boqué N, Martínez JA. Healthy properties of proanthocyanidins. BioFactors 2010; 36: 159-168.

39. Marie A. Oligomeric Proanthocyanidin Complexes: History, Structure, and Phytopharmaceutical Applications. Altern Med Rev 2000; 5: 144-151

40. Shahat AA, Cos P, De Bruyne T, Apers S, Hammouda FM, Ismail SI, et al. Antiviral and Antioxidant Activity of Flavonoids and Proanthocyanidins from Crataegus sinaica. Planta Med 2002; 68: 539-541.

41. Zhang M, Wu Q, Chen Y, Duan M, Tian G, Deng X, et al. Inhibition of proanthocyanidin 
A2 on porcine reproductive and respiratory syndrome virus replication in vitro. PloS One 2018; 13: e0193309.

42. Gallina L, Dal Pozzo F, Galligioni V, Bombardelli E, Scagliarini A. Inhibition of viral RNA synthesis in canine distemper virus infection by proanthocyanidin A2. Antivir Res 2011; 92: 447-452.

43. Zhuang M, Jiang H, Suzuki Y, Li X, Xiao P, Tanaka T, et al. Procyanidins and butanol extract of Cinnamomi Cortex inhibit SARS-CoV infection. Antivir Res 2009; 82: 73-81.

44. Ratia K, Saikatendu KS, Santarsiero BD, Barretto N, Baker SC, Stevens RC, et al. Severe acute respiratory syndrome coronavirus papain-like protease: Structure of a viral deubiquitinating enzyme. PNAS 2006; 103: 5717-5722.

45. Ratia K, Kilianski A, Baez-Santos YM, Baker SC, Mesecar A. Structural Basis for the Ubiquitin-Linkage Specificity and deISGylating Activity of SARS-CoV Papain-Like Protease. PLoS Pathog 2014; 10: e1004113.

46. Calistri A, Munegato D, Carli I, Parolin C, Palù G. The Ubiquitin-Conjugating System: Multiple Roles in Viral Replication and Infection. Cells 2014; 3: 386-417.

47. Barretto N, Jukneliene D, Ratia K, Chen Z, Mesecar AD, Baker SC. The Papain-Like Protease of Severe Acute Respiratory Syndrome Coronavirus Has Deubiquitinating Activity. J Virol 2005; 79: 15189-15198.

48. Lindner HA, Fotouhi-Ardakani N, Lytvyn V, Lachance P, Sulea T, Ménard R. The Papain-Like Protease from the Severe Acute Respiratory Syndrome Coronavirus Is a Deubiquitinating Enzyme. J Virol 2005; 79: 15199-151208.

49. Amin SA, Ghosh K, Gayen S, Jha T. Chemical-informatics approach to COVID-19 drug discovery: Monte Carlo based QSAR, virtual screening and molecular docking study of some in-house molecules as papainlike protease (PLpro) inhibitors, J Biomol Struct Dyn. DOI:10.1080/07391102.2020.1780946

50. Kolodziejczyk-Czepas J, Czepas J. Rhaponticin as an anti-inflammatory component of rhubarb: a minireview of the current state of the art and prospects for future research. Phytochem Rev 2019; 18: 1375-1386.

51. Gelpi J, Hospital A, Goñi R, Orozco M. Molecular dynamics simulations: advances and applications. Adv Appl Bioinform Chem 2015; 8: 37-47.

52. Mohammad T, Arif K, Alajmi MF, Hussain A, Islam A, Rehman MT, et al. Identification of high-affinity inhibitors of pyruvate dehydrogenase kinase-3: towards therapeutic management of cancer. J Biomol Struct Dyn 2020; 1-9. doi:10.1080/07391102.2020.1711810

53. Shamsi A, Mohammad T, Anwar S, AlAjmi MF, Hussain A, Rehman MT, et al. Glecaprevir and Maraviroc are high-affinity inhibitors of SARS-CoV-2 main protease: possible implication in COVID-19 therapy. Biosci Rep 2020; 40: BSR20201256.

54. Rodier F, Bahadur RP, Chakrabarti P, Janin J. Hydration of protein-protein interfaces. Proteins 2005; 60: 36-45.

55. Guan L, Yang H, Cai Y, Sun L, Di P, Li W, et al. ADMET-score-a comprehensive scoring function for evaluation of chemical drug-likeness. Med Chem Comm 2019; 10: 148-57. 
Table 1: Extra precision (XP) molecular docking and Prime/MM-GBSA scores of SARS-CoV-2 Mpro and PLpro with the most promising natural compounds

\begin{tabular}{|c|c|c|c|c|c|c|}
\hline $\begin{array}{l}\text { S. } \\
\text { No. }\end{array}$ & Name of compound & $\begin{array}{c}\text { Docking } \\
\text { score } \\
\text { (kcal/mol) } \\
\end{array}$ & $\begin{array}{c}\text { Glide g- } \\
\text { score } \\
\text { (kcal/mol) }\end{array}$ & $\begin{array}{c}\text { Glide e- } \\
\text { model } \\
(\mathrm{kcal} / \mathrm{mol})\end{array}$ & $\begin{array}{c}\text { Glide } \\
\text { energy } \\
\text { (kcal/mol) }\end{array}$ & $\begin{array}{c}\text { Prime/ } \\
\text { MM-GBSA } \\
\text { (kcal/mol) }\end{array}$ \\
\hline \multicolumn{7}{|c|}{ 3CLpro } \\
\hline 1. & Polymyxin B & -10.704 & -10.728 & -70.234 & -97.031 & -54.49 \\
\hline 2. & Amikacin & -10.599 & -10.771 & -97.087 & -73.774 & -78.47 \\
\hline 3. & Proanthocyanidins & -10.566 & -10.566 & -76.124 & -61.348 & -89.98 \\
\hline 4. & Typhaneoside & -10.515 & -10.590 & -89.512 & -70.493 & -54.32 \\
\hline 5. & $\begin{array}{l}\text { Kaempferol-3-O- } \\
\text { glucorhmanoside }\end{array}$ & -9.442 & -9.517 & -71.852 & -61.614 & -37.95 \\
\hline 6. & Complanatuside & -9.103 & -9.108 & -87.060 & -69.649 & -57.17 \\
\hline \multicolumn{7}{|c|}{ PLpro } \\
\hline 1. & Rhapontin & -10.022 & -10.022 & -58.158 & -43.833 & -61.39 \\
\hline 2. & $\begin{array}{l}\text { Secoisolariciresinol } \\
\text { diglucoside }\end{array}$ & -9.374 & -9.374 & -71.807 & -56.090 & -55.25 \\
\hline 3. & Eriocitrin & -8.677 & -8.688 & -70.421 & -53.409 & -58.21 \\
\hline 4. & Sophoricoside & -8.066 & -8.140 & -60.022 & -45.249 & -52.12 \\
\hline 5. & Nadide & -5.629 & -5.630 & -62.341 & -52.532 & -49.14 \\
\hline 6. & $(+)$-Catechin & -5.006 & -5.006 & -41.984 & -33.828 & -36.03 \\
\hline
\end{tabular}

The compounds are arranged on the basis of Docking scores. The compounds shown in bold were selected for molecular dynamics simulation. 
Table 2: Molecular interaction between SARS-CoV-2 Mpro and PLpro proteins with their respective inhibitors

\begin{tabular}{|c|c|c|c|c|c|}
\hline $\begin{array}{l}\text { Hydrogen } \\
\text { bonds }\end{array}$ & $\begin{array}{c}\text { Hydrophobic } \\
\text { interactions }\end{array}$ & $\begin{array}{c}\text { Other residues } \\
\text { making van der } \\
\text { Waals' interactions }\end{array}$ & $\begin{array}{c}\text { Docking } \\
\text { energy, } \Delta G \\
(\mathrm{kcal} / \mathrm{mol}) \\
\end{array}$ & $\begin{array}{c}\text { Docking } \\
\text { affinity, } K_{\mathrm{d}} \\
\left(\mathbf{M}^{-1}\right) \\
\end{array}$ & $\begin{array}{c}\text { Ligand } \\
\text { efficiency, LE } \\
\text { (kcal/mol) } \\
\end{array}$ \\
\hline \multicolumn{6}{|c|}{ Mpro-Proanthocyanidin complex } \\
\hline $\begin{array}{l}\text { Asn142, } \\
\text { Cys145, } \\
\text { His163, } \\
\text { Glu166, } \\
\text { Gln189, } \\
\text { Thr190 }\end{array}$ & Hie41 & $\begin{array}{l}\text { Phe140, Leu141, } \\
\text { Gly143, Ser144, } \\
\text { His164, Met165, } \\
\text { Leu167, Pro168, } \\
\text { Hie172, Val186, } \\
\text { Asp187, Arg188, } \\
\text { Ala191, Gln192 }\end{array}$ & -10.566 & $5.62 \times 10^{7}$ & -0.153 \\
\hline \multicolumn{6}{|c|}{ PLpro-Rhapontin complex } \\
\hline $\begin{array}{l}\text { Trp106, } \\
\text { Gly271, } \\
\text { Hie272, } \\
\text { Asp286 }\end{array}$ & Hie272 & $\begin{array}{l}\text { Lys105, Asn109, } \\
\text { Cys111, Leu162, } \\
\text { Gln269, Cys270, } \\
\text { Tyr273, Gly287, } \\
\text { Ala288, Leu289 }\end{array}$ & -10.022 & $2.24 \times 10^{7}$ & -0.186 \\
\hline
\end{tabular}

The residues shown in bold are catalytic residues. Ligand efficiency (LE) was calculated using the relation, $\mathrm{LE}=\Delta G / \mathrm{N}$; where $\mathrm{N}$ is the number of non-hydrogen atoms in the ligand. 
Table 3: Physicochemical and ADMET (Adsorption, Distribution, Metabolism, Excretion, and Toxicity) properties of Proanthocyanidin and Rhapontin

\begin{tabular}{l|l|l|l}
\hline Properties & Proanthocyanidin & Rhapontin & Acceptable range \\
\hline Mol_MW & 594.528 & 420.415 & $150-750$ \\
SASA & 875.847 & 712.813 & $300-1000$ \\
FOSA & 34.859 & 226.616 & $0-750$ \\
FISA & 436.944 & 270.826 & $7-330$ \\
PISA & 404.044 & 215.371 & $0-450$ \\
WPSA & 0 & 0 & $0-175$ \\
Volume & 1616.909 & 1263.322 & - \\
donorHB & 10 & 6 & 5 \\
accptHB & 12 & 12 & 10 \\
Rotatable bonds & 12 & 13 & 10 \\
QPpolrz & 54.708 & 38.114 & $13-70$ \\
QPlogPC16 & 21.708 & 14.803 & $4-18$ \\
QPlogPoct & 41.236 & 27.696 & $8-35$ \\
QPlogPw & 31.022 & 21.989 & $4-45$ \\
QPlogPo/w & 0.486 & 0.268 & -2 to 6.5 \\
QPlogS & -4.95 & -3.128 & -6.5 to 0.5 \\
CIQPlogS & -7.284 & -3.83 & -6.5 to 0.5 \\
QPlogHERG & -7.332 & -5.911 & Concern below -5 \\
QPPCaco & 0.712 & 26.771 & $<25$ poor, $>500$ great \\
QPlogBB & -5.194 & -3.092 & -3 to 1.2 \\
QPPMDCK & 0.196 & 9.882 & $<25$ poor, $>500$ great \\
QPlogKp & -6.997 & -4.504 & -8 to -1 \\
QPlogKhsa & -0.338 & -0.738 & -1.5 to 1.5 \\
Oral absorption $(\%)$ & 0 & 40 & $<25 \%$ poor, $>80 \%$ great \\
\hline
\end{tabular}

Mol_MW: Molecular weight; SASA: Solvent accessible surface area; FOSA: Hydrophobic component of SASA; FISA: Hydrophilic component of SASA; PISA: Polar component of SASA; WPSA: Weakly polar component of SASA; donorHB: Hydrogen bond donors; accptHB: Hydrogen bond acceptor; QPpolrz: Polarizability; QPlogPC16: Hexadecane/gas partition coefficient; QPlogPoct: Octanol/gas partition coefficient; QPlogPw: Water/gas partition coefficients; QP $\log \mathrm{Po} / \mathrm{w}$ : Octanol/water partition coefficients; QPlogS: Solubility; CIQPlogS: Conformation-dependent solubility; QPlogHERG: blockage of HERG K+ channels; QPPCaco: CACO permeability; QPlogBB: bloor/brain partition coefficient; QPPMDCK: MDCK cell permeability; QPlogKp: Skin permeability; QPlogKhsa: Binding to human serum albumin 


\section{Supplementary Table S1: Standard precision (SP) molecular docking of SARS-CoV-2 Mpro with top 50 natural compounds from L1400 library of Selleck Inc.}

\begin{tabular}{|c|c|c|c|c|c|c|}
\hline $\begin{array}{l}\text { S. } \\
\text { No. }\end{array}$ & $\begin{array}{l}\text { Name of } \\
\text { compounds }\end{array}$ & $\begin{array}{l}\text { Nature of the } \\
\text { compound }\end{array}$ & $\begin{array}{c}\text { Docking } \\
\text { score } \\
\text { (kcal/mol) }\end{array}$ & $\begin{array}{c}\text { Glide g- } \\
\text { score } \\
\text { (kcal/mol) }\end{array}$ & $\begin{array}{c}\text { Glide e- } \\
\text { model } \\
\text { (kcal/mol) }\end{array}$ & $\begin{array}{c}\text { Glide } \\
\text { energy } \\
\text { (kcal/mol) }\end{array}$ \\
\hline 1. & Polymyxin B & Antibiotic & -11.212 & -11.236 & -152.814 & -88.071 \\
\hline 2. & Complanatuside & Flavonoid & -8.667 & -8.672 & -103.861 & -74.223 \\
\hline 3. & $\begin{array}{l}\text { Kaempferol-3-O- } \\
\text { glucorhaMnoside }\end{array}$ & Flavonoid & -8.642 & -8.717 & -93.836 & -67.147 \\
\hline 4. & Proanthocyanidin & Polyphenols & -8.513 & -8.513 & -91.259 & -61.562 \\
\hline 5. & Typhaneoside & $\begin{array}{l}\text { Flavonoid-3-o- } \\
\text { glycosides }\end{array}$ & -8.350 & -8.425 & -95.466 & -65.687 \\
\hline 6. & Amikacin & Antibiotic & -8.236 & -8.408 & -124.058 & -75.963 \\
\hline 7. & Hederacoside D & Saponins & -7.915 & -7.915 & -98.653 & -70.812 \\
\hline 8. & Thymopentin & Immunostimulant & -7.741 & -8.030 & -97.893 & -62.710 \\
\hline 9. & Specnuezhenide & $\begin{array}{l}\text { Ingredients of } \\
\text { chinese medicine }\end{array}$ & -7.686 & -7.687 & -92.232 & -65.735 \\
\hline 10. & $\begin{array}{l}1,2,3,4,6-\mathrm{O}- \\
\text { Pentagalloylglucose }\end{array}$ & Ester of glucose & -7.644 & -8.188 & -107.694 & -76.130 \\
\hline 11. & Parishin A & Natural product & -7.628 & -7.628 & -100.862 & -70.823 \\
\hline 12. & Isepamicin & $\begin{array}{l}\text { Aminoglycoside } \\
\text { antibiotic }\end{array}$ & -7.610 & -7.792 & -101.710 & -55.975 \\
\hline 13. & Netilmicin & $\begin{array}{l}\text { Aminoglycoside } \\
\text { antibiotics }\end{array}$ & -7.559 & -8.328 & -103.437 & -57.087 \\
\hline 14. & Azlocillin & $\begin{array}{l}\text { Acylampicillin } \\
\text { antibiotic }\end{array}$ & -7.516 & -7.516 & -76.238 & -54.954 \\
\hline 15. & Naringenin & Flavonoid & -7.270 & -7.318 & -55.678 & -38.884 \\
\hline 16. & Sarafloxacin & $\begin{array}{l}\text { Quinolone } \\
\text { antibiotic }\end{array}$ & -7.256 & -7.297 & -70.159 & -45.423 \\
\hline 17. & $\begin{array}{l}\text { 10-Hydroxy- } \\
\text { camptothecin }\end{array}$ & Indole alkaloid & -7.141 & -7.159 & -64.678 & -46.153 \\
\hline 18. & Cinchonine & Alkaloid & -7.073 & -7.108 & -59.588 & -40.748 \\
\hline 19. & Daurisoline & Alkaloid & -6.960 & -7.227 & -88.021 & -56.676 \\
\hline 20. & Paclitaxel & $\begin{array}{l}\text { Anticancer } \\
\text { medicine }\end{array}$ & -6.947 & -6.951 & -83.816 & -60.998 \\
\hline 21. & Lycorine & Alkaloid & -6.883 & -6.966 & -59.820 & -39.557 \\
\hline 22. & Oroxin B & Flavonoid & -6.879 & -6.898 & -85.265 & -57.345 \\
\hline 23. & Tosufloxacin & $\begin{array}{l}\text { Fluoroquinolone } \\
\text { antibiotic }\end{array}$ & -6.844 & -6.853 & -66.704 & -44.610 \\
\hline 24. & Halofuginone & $\begin{array}{l}\text { Alkaloid } \\
\text { derivative }\end{array}$ & -6.828 & -6.854 & -70.026 & -47.192 \\
\hline 25. & Dracohodin & $\begin{array}{l}\text { Extracted from } \\
\text { Dragon blood }\end{array}$ & -6.790 & -6.808 & -57.552 & -39.256 \\
\hline 26. & Galangin & Flavonoid & -6.743 & -6.862 & -51.265 & -37.576 \\
\hline 27. & Tigecycline & Antibiotic & -6.704 & -7.039 & -90.316 & -60.082 \\
\hline 28. & Sparteine & Alkaloid & -6.685 & -6.733 & -57.351 & -34.457 \\
\hline 29. & Mezlocillin & $\begin{array}{l}\text { Penicillin } \\
\text { antibiotic }\end{array}$ & -6.649 & -6.649 & -76.826 & -59.164 \\
\hline 30. & Nuciferine & Alkaloid & -6.509 & -6.602 & -53.015 & -34.684 \\
\hline
\end{tabular}




\begin{tabular}{|c|c|c|c|c|c|}
\hline Icariin & Flavonoid & -6.494 & -6.501 & -74.989 & -59.878 \\
\hline Epigoitrin & Alkaloid & -6.457 & -6.457 & -32.161 & -22.789 \\
\hline Isosakuranetin & Flavanone & -6.456 & -6.503 & -51.432 & -36.387 \\
\hline Tubeimoside I & Terpenoid & -6.438 & -6.438 & -72.261 & -59.102 \\
\hline Pectolinarin & $\begin{array}{l}\text { Natural anti- } \\
\text { inflammatory } \\
\text { compound }\end{array}$ & -6.429 & -6.438 & -82.760 & -62.673 \\
\hline Stylopine & Alkaloid & -6.286 & -6.440 & -57.389 & -38.297 \\
\hline Norisoboldine & Alkaloid & -6.278 & -6.365 & -56.265 & -37.636 \\
\hline Docetaxel & $\begin{array}{l}\text { Anticancerous } \\
\text { medicine }\end{array}$ & -6.166 & -6.171 & -73.512 & -55.484 \\
\hline Forchlorfenuron & $\begin{array}{l}\text { Plant growth } \\
\text { regulator }\end{array}$ & -6.147 & -6.147 & -48.372 & -36.017 \\
\hline Scutellarein & Flavone & -6.132 & -6.253 & -52.378 & -37.860 \\
\hline $\begin{array}{l}\text { (-)-Epicatechin } \\
\text { gallate }\end{array}$ & Flavonoid & -6.084 & -6.261 & -72.482 & -54.519 \\
\hline Sparfloxacin & $\begin{array}{l}\text { Fluoroquinolones } \\
\text { (antibiotic) }\end{array}$ & -5.995 & -6.012 & -57.746 & -41.640 \\
\hline Piperlongumine & $\begin{array}{l}\text { Natural product } \\
\text { (long pepper) }\end{array}$ & -5.985 & -5.985 & -51.366 & -38.708 \\
\hline Myricetin & Flavonoid & -5.935 & -6.037 & -56.332 & -43.530 \\
\hline Matrine & Alkaloid & -5.853 & -5.855 & -47.772 & -32.797 \\
\hline Trecator & Antibiotic & -5.845 & -5.846 & -35.597 & -26.116 \\
\hline Neomycin & $\begin{array}{l}\text { Aminoglycoside } \\
\text { antibiotic }\end{array}$ & -5.816 & -7.019 & -103.362 & -57.295 \\
\hline Biochanin A & Monoflavonoid & -5.754 & -5.829 & -52.431 & -38.873 \\
\hline Liensinine & Alkaloid & -5.604 & -5.816 & -77.632 & -52.888 \\
\hline Kaempferide & Flavonol & -5.424 & -5.534 & -63.245 & -49.275 \\
\hline
\end{tabular}




\section{Supplementary Table S2: Standard precision (SP) molecular docking of SARS-CoV-2 PLpro with top 50 natural compounds from L1400 library of Selleck Inc.}

\begin{tabular}{|c|c|c|c|c|c|c|}
\hline $\begin{array}{l}\text { S. } \\
\text { No. }\end{array}$ & Name of compounds & $\begin{array}{l}\text { Nature of } \\
\text { compounds }\end{array}$ & $\begin{array}{c}\text { Docking } \\
\text { score } \\
\text { (kcal/mol) } \\
\end{array}$ & $\begin{array}{c}\text { Glide g- } \\
\text { score } \\
(\mathrm{kcal} / \mathrm{mol}) \\
\end{array}$ & $\begin{array}{c}\text { Glide e- } \\
\text { model } \\
(\mathrm{kcal} / \mathrm{mol}) \\
\end{array}$ & $\begin{array}{c}\text { Glide } \\
\text { energy } \\
\text { (kcal/mol) }\end{array}$ \\
\hline 1. & $\begin{array}{l}\text { Secoisolariciresinol } \\
\text { diglucoside }\end{array}$ & $\begin{array}{l}\text { Antioxidant } \\
\text { phytoestrogen }\end{array}$ & -6.973 & -6.973 & -76.181 & -59.119 \\
\hline 2. & Sophoricoside & Isoflavone & -6.403 & -6.477 & -55.728 & -45.598 \\
\hline 3. & Eriocitrin & $\begin{array}{l}\text { Flavanone-7-O- } \\
\text { glycoside }\end{array}$ & -6.204 & -6.215 & -75.626 & -55.826 \\
\hline 4. & $(+)$-Catechin & Natural phenol & -6.186 & -6.186 & -46.521 & -34.584 \\
\hline 5. & Rhapontin & Glucoside & -6.182 & -6.182 & -57.016 & -46.366 \\
\hline 6. & Nadide & Dinucleotide & -6.124 & -6.126 & -71.067 & -48.557 \\
\hline 7. & Benzoylpaeoniflorin & Natural product & -5.997 & -5.997 & -61.594 & -51.320 \\
\hline 8. & Cytisine & Alkaloid & -5.980 & -5.981 & -34.385 & -24.702 \\
\hline 9. & Naringenin & Flavonoid & -5.957 & -6.005 & -44.403 & -31.896 \\
\hline 10. & Yohimbine & Alkaloid & -5.875 & -5.938 & -39.950 & -31.068 \\
\hline 11. & Apigenin & Flavone & -5.866 & -5.967 & -41.514 & -31.231 \\
\hline 12. & 4-Hydroxyquinazoline & $\begin{array}{l}\text { Heterocyclic } \\
\text { compound }\end{array}$ & -5.842 & -5.890 & -33.189 & -23.924 \\
\hline 13. & (-)-Epicatechin & Natural phenol & -5.830 & -5.830 & -44.749 & -33.664 \\
\hline 14. & Norcantharidin & $\begin{array}{l}\text { Derivative of } \\
\text { natural product }\end{array}$ & -5.789 & -5.789 & -33.507 & -25.087 \\
\hline 15. & (+)-Gallocatechin & Flavan-3-ol & -5.782 & -5.800 & -46.411 & -34.379 \\
\hline 16. & Isatin & Indole & -5.716 & -5.716 & -31.563 & -22.743 \\
\hline 17. & Oxyresveratrol & Stillbenoid & -5.715 & -5.726 & -41.389 & -30.426 \\
\hline 18. & Phloretin & Natural phenol & -5.691 & -5.894 & -46.470 & -33.988 \\
\hline 19. & N-Ethylmaleimide & $\begin{array}{l}\text { Organic } \\
\text { compound }\end{array}$ & -5.676 & -5.676 & -28.492 & -21.348 \\
\hline 20. & (-)-Epicatechin gallate & Flavonoid & -5.669 & -5.846 & -61.209 & -50.003 \\
\hline 21. & Neohesperidin & $\begin{array}{l}\text { Flavanone } \\
\text { glycoside }\end{array}$ & -5.627 & -5.638 & -57.750 & -44.917 \\
\hline 22. & Benzoyleneurea & Quinazolinedione & -5.624 & -5.640 & -32.964 & -24.244 \\
\hline 23. & 2-Benzoxazolinone & Phytoantpicin & -5.623 & -5.636 & -31.920 & -23.118 \\
\hline 24. & Kynurenic acid & $\begin{array}{l}\text { Metabolic product } \\
\text { of L-Tryptophan }\end{array}$ & -5.607 & -5.607 & -35.762 & -24.631 \\
\hline 25. & Eriodictyol & Flavonoid & -5.588 & -5.636 & -46.142 & -35.240 \\
\hline 26. & Rhapontigenin & Stillbenoid & -5.578 & -5.589 & -41.829 & -32.264 \\
\hline 27. & $\begin{array}{l}\text { 7-Hydroxy-3,4- } \\
\text { dihydrocarbostyril }\end{array}$ & $\begin{array}{l}\text { Synthetic } \\
\text { quinlonine } \\
\text { compound }\end{array}$ & -5.547 & -5.550 & -34.225 & -24.752 \\
\hline 28. & Echinatin & Licorice extract & -5.545 & -5.721 & -42.855 & -32.305 \\
\hline 29. & Lawsone & Naphthoquinone & -5.528 & -5.528 & -34.827 & -24.365 \\
\hline 30. & Indole-3-acetic acid & $\begin{array}{l}\text { Plant hormone } \\
\text { Intermediate }\end{array}$ & -5.515 & -5.516 & -35.927 & -23.890 \\
\hline 31. & Dihydrothymine & $\begin{array}{l}\text { product of } \\
\text { thymine }\end{array}$ & -5.492 & -5.492 & -30.993 & -23.351 \\
\hline 32. & Sophocarpine & Natural product & -5.475 & -5.477 & -36.052 & -27.078 \\
\hline
\end{tabular}


33. Umbelliferone

34. Oxindole

35. Gallic acid

36. Daphnetin

37. Acetophenone

38. Plumbagin

39. Carvacrol

40. 7-Hydroxyflavone

41. Daidzein

42. Apatinib

43. Xanthurenic Acid

44. Paeoniflorin

45. Isopsoralen

46. 4-Isopropyl benzaldehyde

47. Alizarin

48. Thymoquinone

49. Juglone

50. 4-Ketoisophorone
Product of coumarin family

Aromatic

heterocyclic

compound

Phenolic acid

Coumarin

Aromatic ketone

Naphthoquinone

Monoterpene

derivative

Flavonoid

Isoflavone

Tyrosine kinase

inhibitor

Intermediate of

Vitamin B6

Constituent of

herbal medicine

Natural

flurocoumarin

Organic

compound

Organic

compound

Phytochemical

compound

Organic

compound

Component of

saffron $\begin{array}{lll}-5.454 & -5.481 \quad-31.138\end{array}$

$-22.523$

$\begin{array}{llll}-5.451 & -5.451 & -28.739 & -21.204\end{array}$

$\begin{array}{llll}-5.448 & -5.448 & -35.003 & -25.121\end{array}$

$\begin{array}{llll}-5.433 & -5.481 & -31.240 & -23.824\end{array}$

$\begin{array}{llll}-5.418 & -5.418 & -25.837 & -19.858\end{array}$

$\begin{array}{llll}-5.372 & -5.397 & -29.496 & -23.785\end{array}$

$\begin{array}{llll}-5.363 & -5.363 & -28.024 & -21.009\end{array}$

$\begin{array}{llll}-5.360 & -5.394 & -35.378 & -26.261\end{array}$

$\begin{array}{llll}-5.356 & -5.378 & -34.025 & -26.242\end{array}$

$\begin{array}{llll}-5.303 & -5.388 & -52.099 & -43.433\end{array}$

$\begin{array}{llll}-5.295 & -5.507 & -36.791 & -24.876\end{array}$

$\begin{array}{llll}-5.281 & -5.281 & -52.460 & -42.792\end{array}$

$\begin{array}{llll}-5.267 & -5.267 & -28.683 & -21.699\end{array}$

$\begin{array}{llll}-5.257 & -5.257 & -26.971 & -20.429\end{array}$

$\begin{array}{llll}-5.222 & -5.323 & -34.916 & -26.463\end{array}$

$\begin{array}{llll}-5.201 & -5.201 & -29.229 & -22.127\end{array}$

$\begin{array}{llll}-5.198 & -5.241 & -28.479 & -23.189\end{array}$

$\begin{array}{llll}-5.185 & -5.185 & -27.324 & -20.868\end{array}$ 


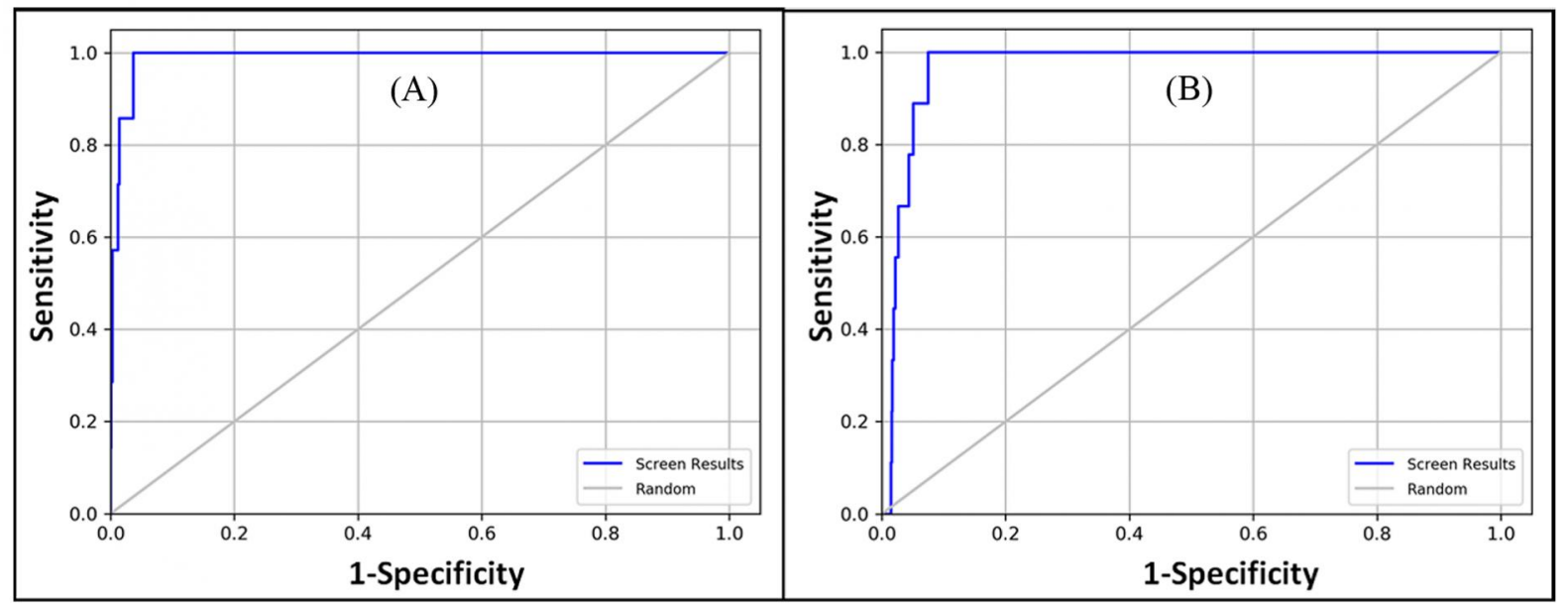

Figure 1: Receiver operating characteristic (ROC) curves of (A) Mpro, and (B) PLpro. The area under curve (AUC) represents sensitivity and specificity of the adopted docking procedure. 


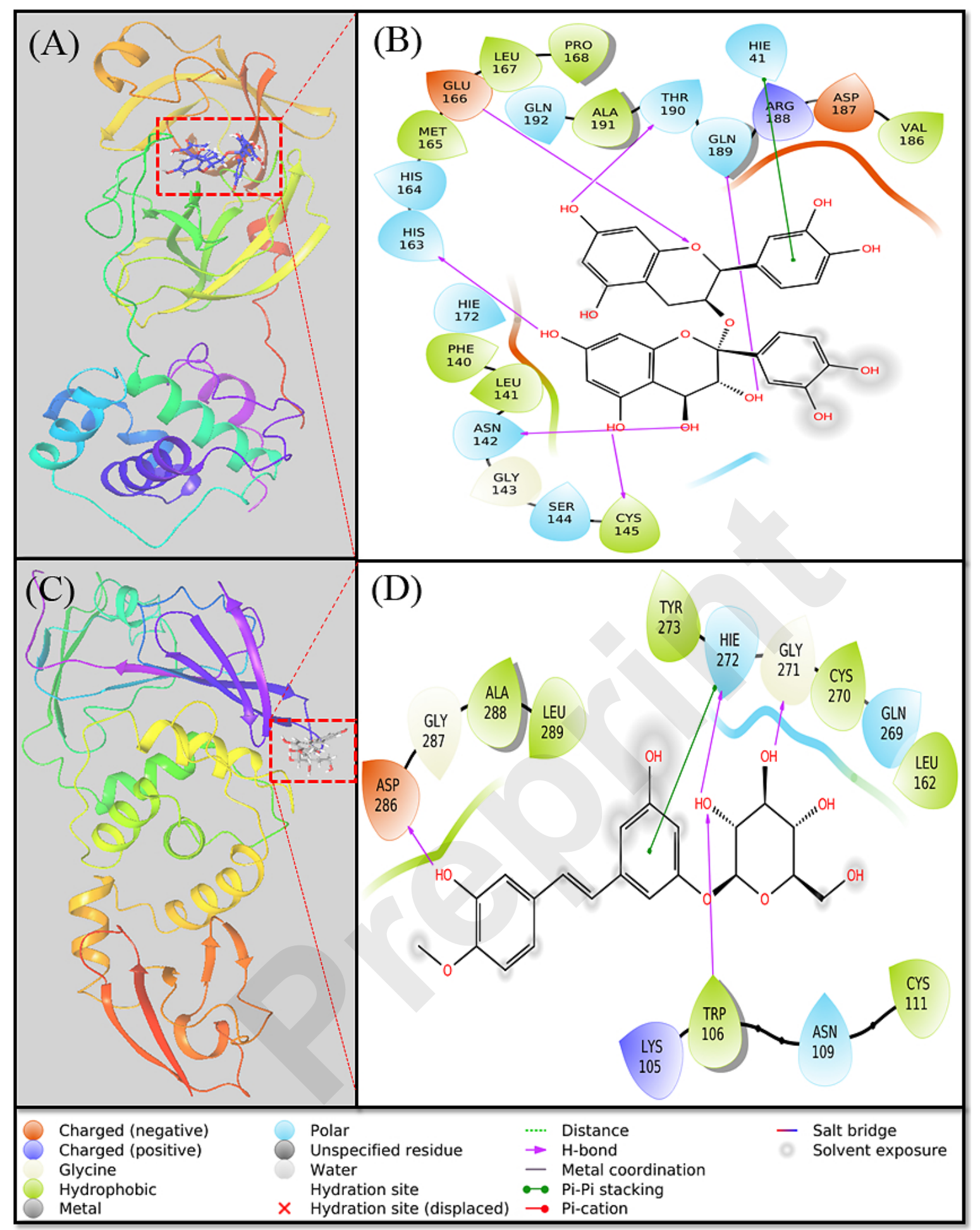

Figure 2: Extra-precision (XP) molecular docking analysis. (A) Binding of Proanthocyanidin to the substrate binding site of Mpro, (B) Molecular interaction between Mpro and Proanthocyanidin, (C) Binding of Rhapontin to the active site of PLpro, and (D) Molecular interaction between PLpro and Rhapontin. 


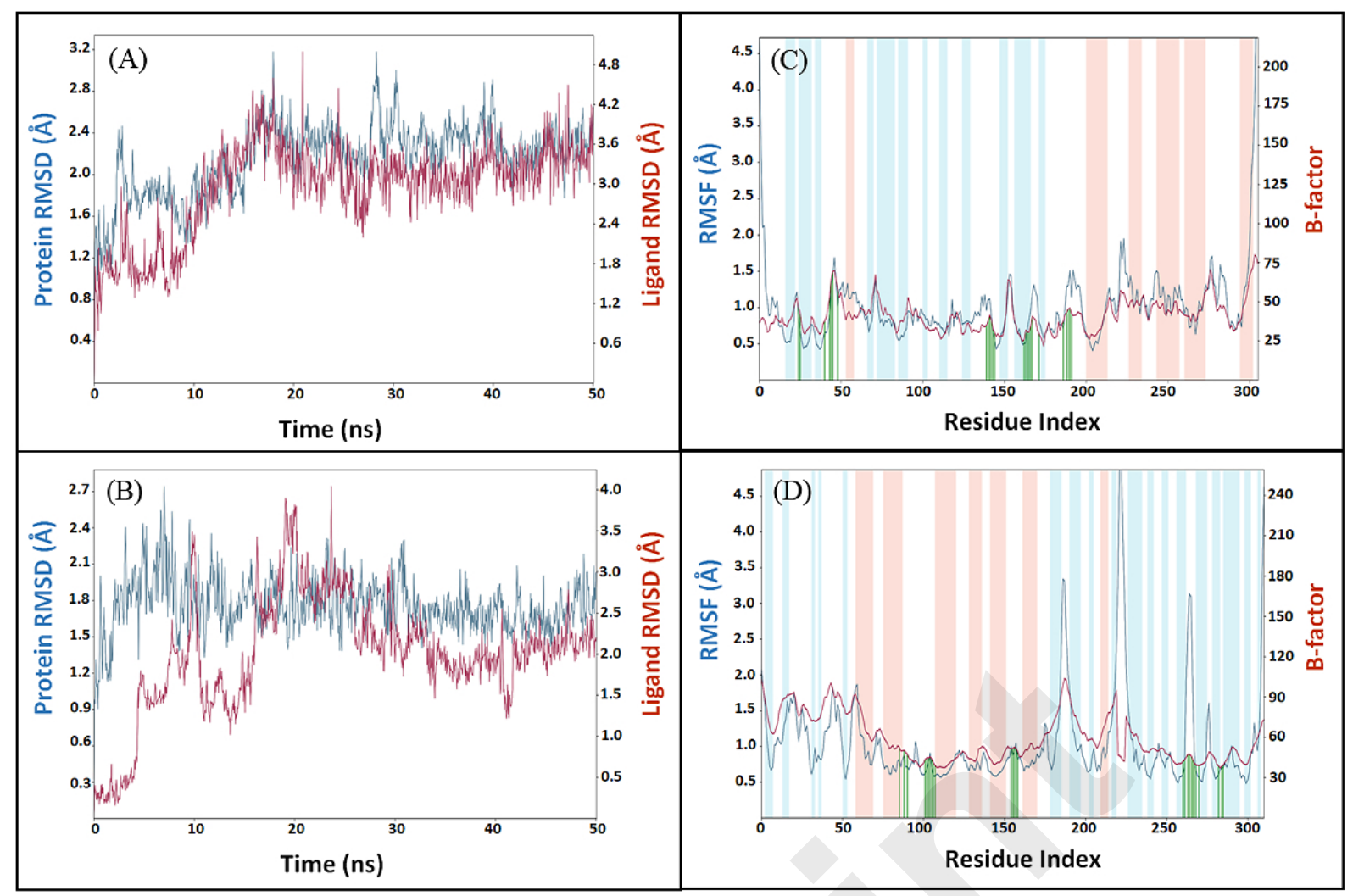

Figure 3: Molecular dynamics (MD) simulation analysis. (A) Root mean square deviation (RMSD) of Mpro alone and in the presence of Proanthocyanidin, (B) RMSD of PLpro alone and in the presence of Rhapontin, (C) Variation in root mean square fluctuation (RMSF) of Mpro in the presence of Proanthocyanidin and comparison with the experimentally determined B-factor during X-ray crystallography, and (D) RMSF of PLpro in the presence of Rhapontin and comparison with the experimentally determined B-factor during X-ray crystallography. In plots $C$ and $D$, the vertical green lines shows the position of amino acid residue involved in the interaction with inhibitor. Also, light brown and teal colors indicate $\alpha-$ helical and $\beta$-sheet regions. 


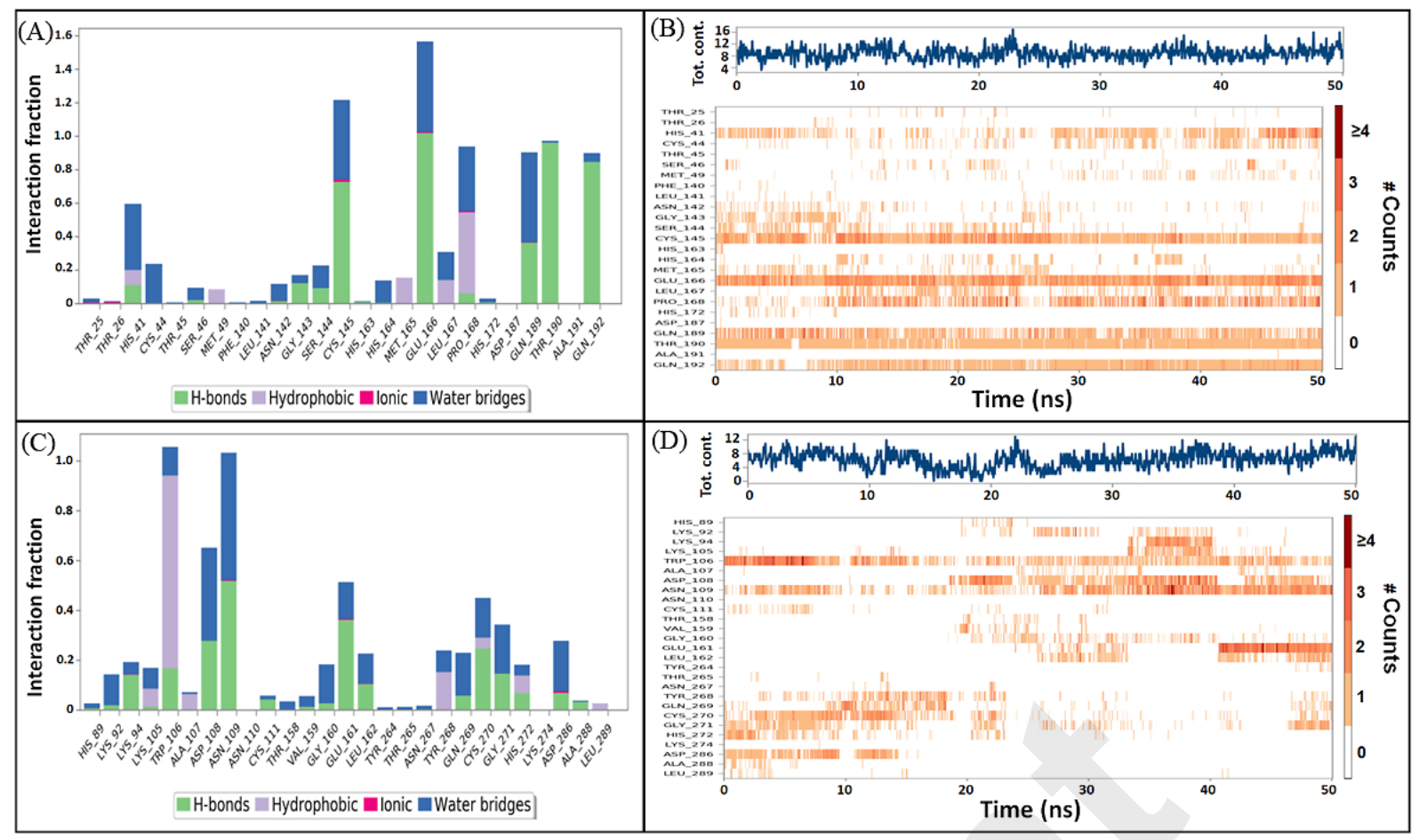

Figure 4: Interaction pattern between proteins and their respective inhibitor as a function of simulation. (A) Involvement of Mpro amino acid residues in forming different types of interaction with Proanthocyanidin, (B) Upper panel: The total number of contacts between Mpro and Proanthocyanidin during simulation. Lower panel: the extent of amino acid residues forming contact with the inhibitor, $(\mathrm{C})$ Involvement of PLpro amino acid residues in forming different types of interaction with Rhapontin, (B) Upper panel: The total number of contacts between PLpro and Rhapontin during simulation. Lower panel: the extent of amino acid residues forming contact with the inhibitor. 

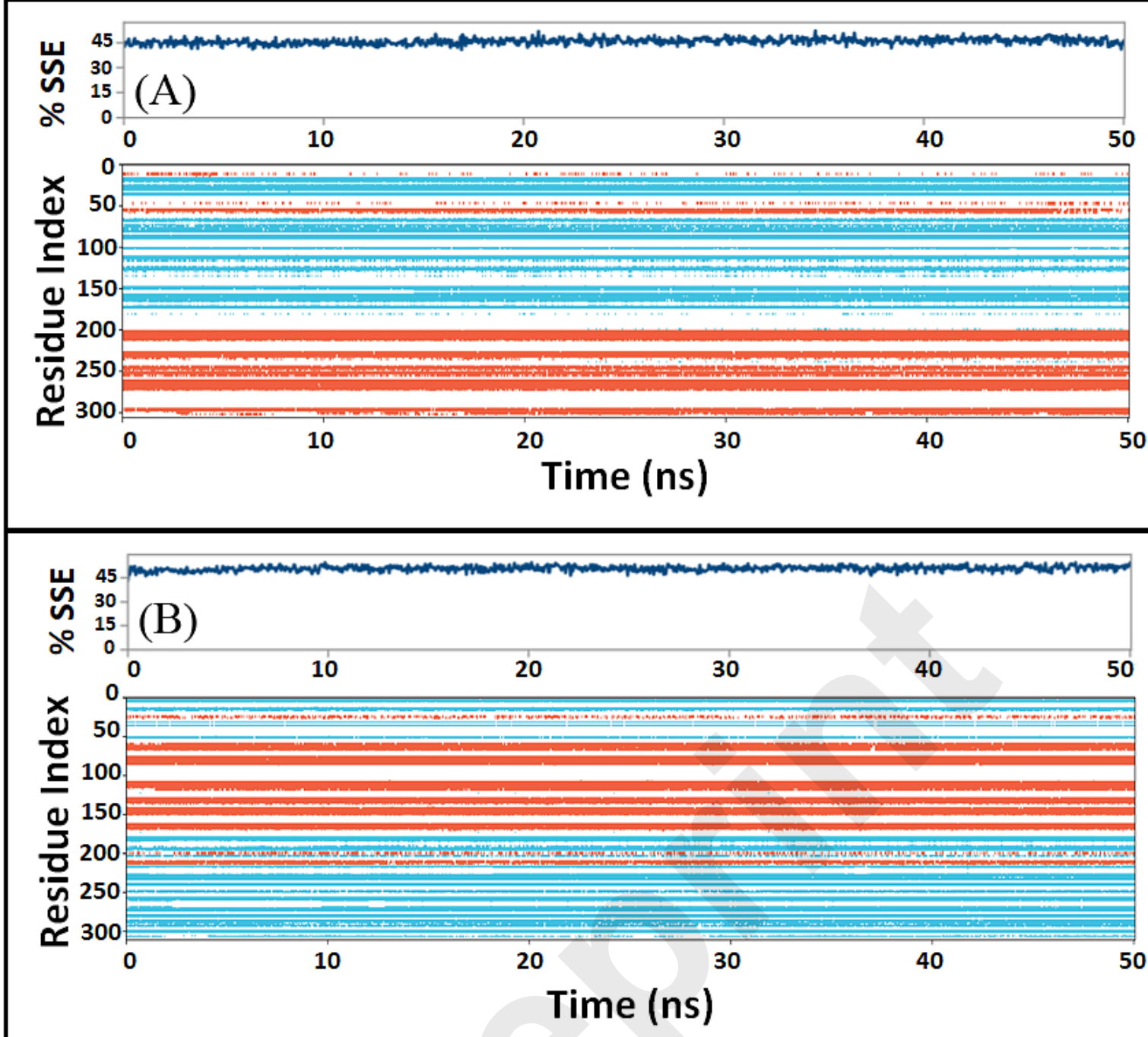

Figure 5: Dependence of secondary structure element (SSE) of during simulation. (A) MproProanthocyanidin complex, and (B) PLpro-Rhapontin complex during simulation. Upper panel: Variation in SSE of proteins as a result of their respective inhibitor binding. Lower panel: Contribution of each amino acid residue in the formation of SSEs wherein $\alpha$-helices are shown in light brown color and $\beta$-sheets are represented in teal color. 


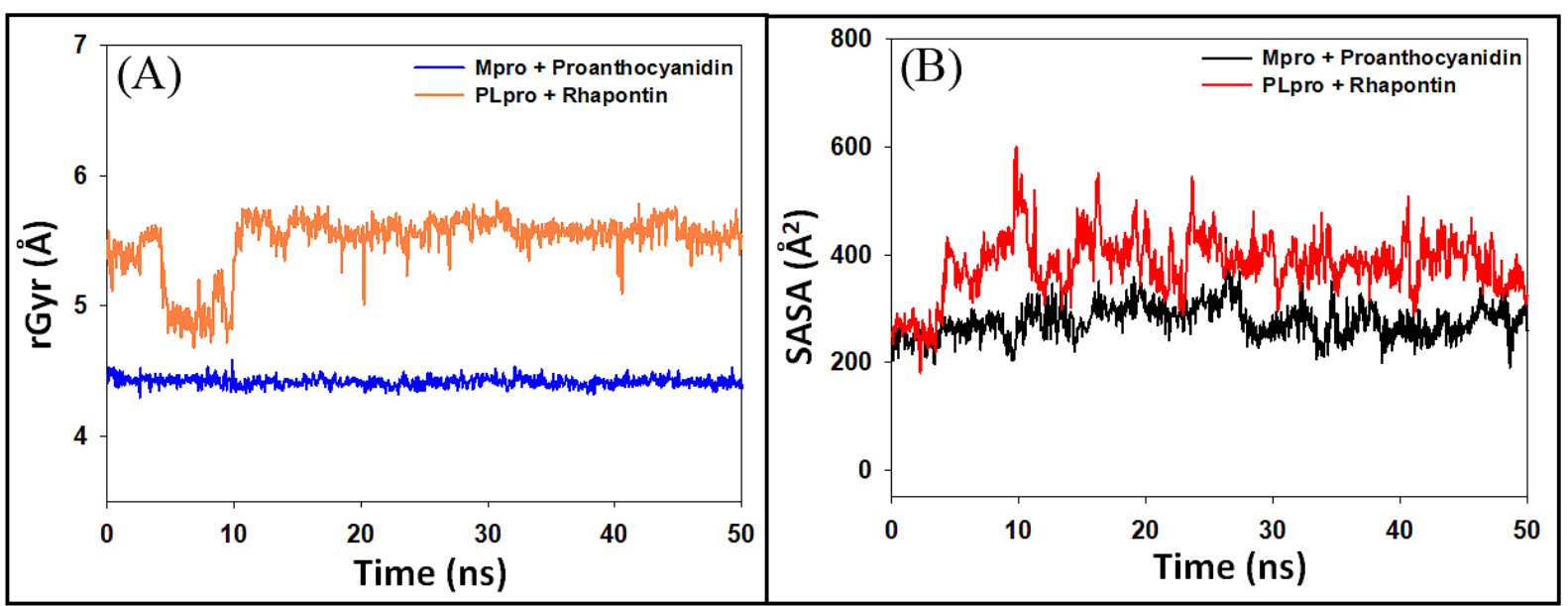

Figure 6: Dependence of $(A)$ radius of gyration ( $r G y r)$ and $(B)$ solvent accessible surface area (SASA) of Mpro-Proanthocyanidin and PLpro-Rhapontin complexes as a function of simulation time. 


\section{RMSD = $1.1564 \AA$}

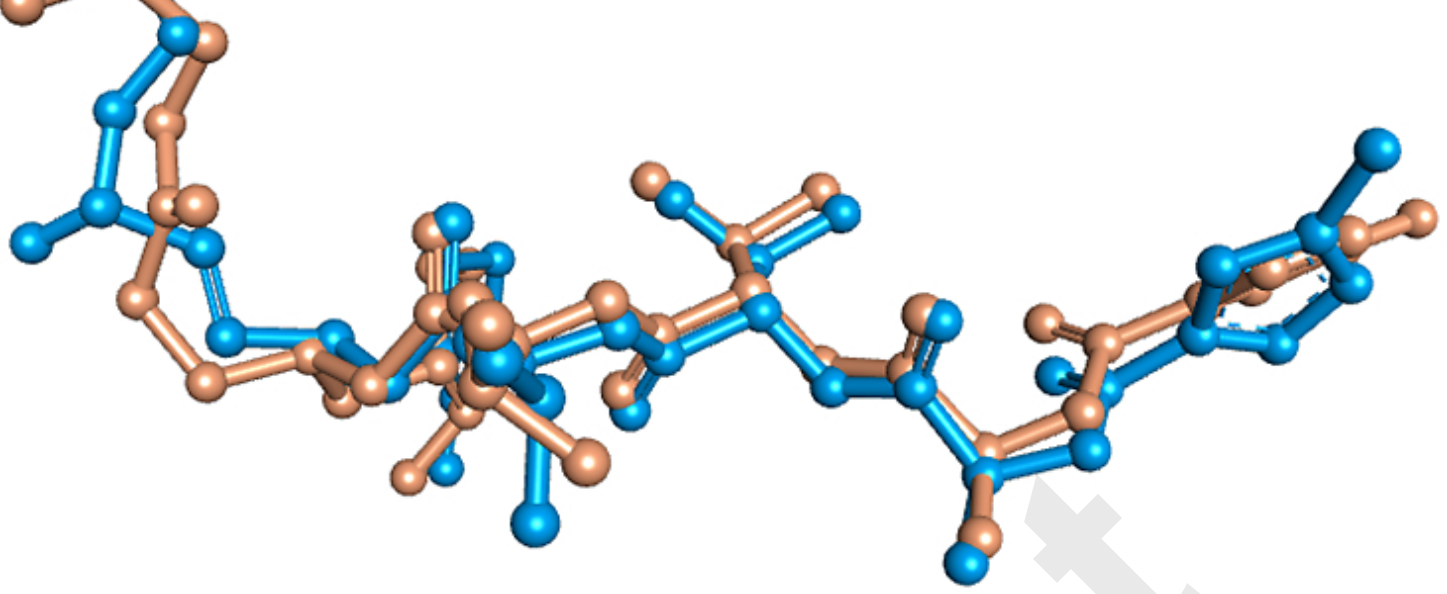

Supplementary Figure S1: Validation of the docking procedure by measuring root mean square deviation (RMSD) between the crystal structure pose and docked pose of ligand (N3) co-crystallized with Mpro. 


\begin{tabular}{|c|c|c|}
\hline SARS-CoV-2 & 1 & 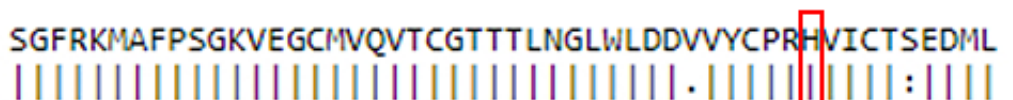 \\
\hline SARS-CoV & 1 & SGFRKMAFPSGKVEGCIVQVTCGTTTLNGLWLDDTVYCPRH HICTAEDIIL \\
\hline SARS-CoV-2 & 51 & 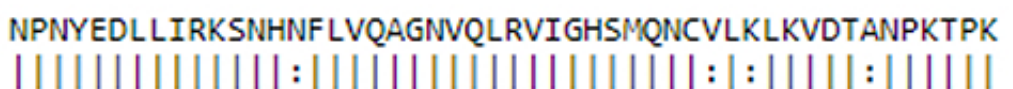 \\
\hline SARS-CoV & 51 & NPNYEDLLIRKSNHSFLVQAGNVQLRVIGHSMQNCLLRLKVDTSNPKTPK \\
\hline SARS-CoV-2 & 101 & 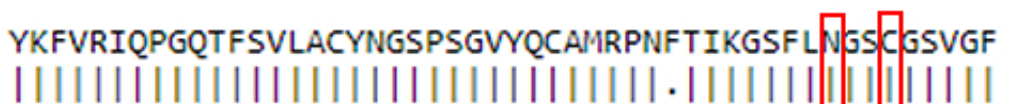 \\
\hline SARS-CoV & 101 & YKFVRIQPGQTFSVLACYNGSPSGVQCAMRPNHTIKGSFLWJSGGSVGF \\
\hline SARS-CoV-2 & 151 & 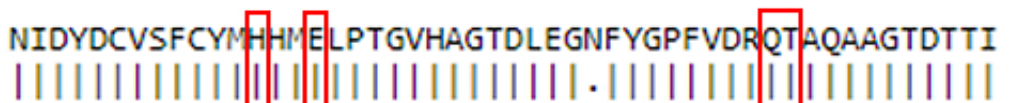 \\
\hline SARS-CoV & 151 & NIDYDCVSFCYM HII'ELPTGVHAGTDLEGKFYGPFVDRQTAQAAGTDTTI \\
\hline SARS-CoV-2 & 201 & $\begin{array}{l}\text { TVNVLAIVLYAAVINGDRWFLNRFTTTLNDFNLVAMKYNYEPLTQDHVDIL } \\
\text { |:||||||||||||||||||||||||||||||||||| }\end{array}$ \\
\hline SARS-CoV & 201 & TLNVLANLYAAVINGDRWFLNRFTTTLNDFNLVAMKYNYEPLTQDHVDIL \\
\hline SARS-CoV-2 & 251 & $\begin{array}{l}\text { GPLSAQTGIAVLDMCASLKELLQNGMNGRTILGSALLEDEFTPFOWVRQC } \\
\text { ||||||||||||||||||||||||||||||||||-|||||||||||||| }\end{array}$ \\
\hline SARS-CoV & 251 & GPLSAQTGIAVLDMCAALKELLQNGMNGRTILGSTILEDEFTPFDVRQC \\
\hline SARS-CoV-2 & 301 & SGVTFQ \\
\hline SARS-CoV & 301 & SGVTFQ \\
\hline
\end{tabular}

Supplementary Figure S2: Pairwise sequence alignment of Mpro derived from SARS-CoV and SARS-CoV-2. The residues inside box are involved in hydrogen bonds and hydrophobic interactions. 


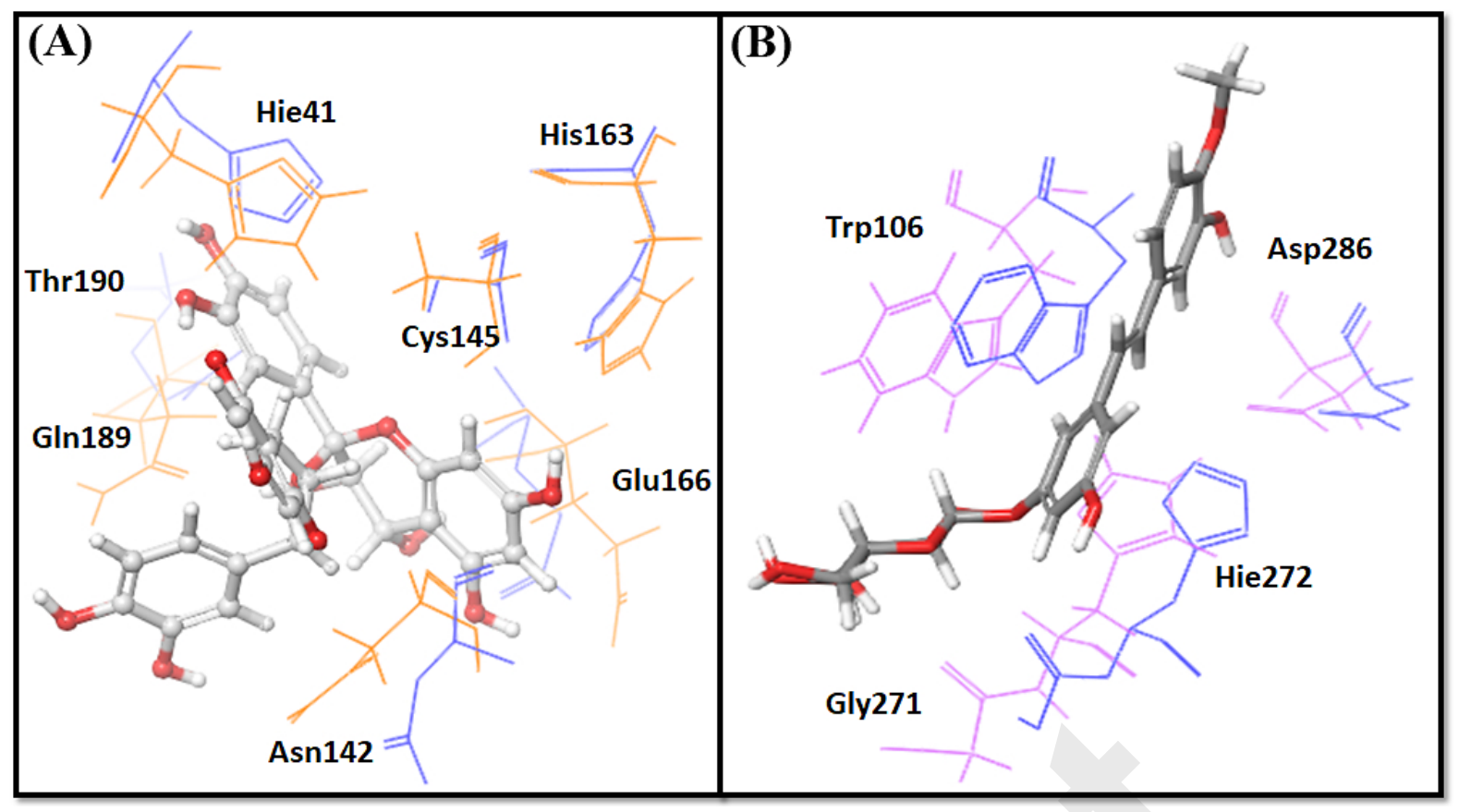

Supplementary Figure S3: Superimposition of (A) SARS-CoV-2 Mpro-Proanthocyanidin, and (B) SARS-CoV-2 PLpro-Rhapontin active sites with that of Mpro and PLpro from SARS$\mathrm{CoV}$. Amino acid residues depicted in purple and golden colors belong to Mpro of SARSCoV and SARS-CoV-2, respectively. Similarly, amino acid residues depicted in magenta and blue colors belong to PLpro of SARS-CoV and SARS-CoV-2, respectively. Amino acid residues depicted in purple and golden belong to Mpro of SARS-CoV and SARS-CoV-2. 


\begin{tabular}{|c|c|c|}
\hline SARS-CoV & 1 & $\begin{array}{l}\text { EVKTIKVFTTVDNTNLHTQLVDMSMTYGQQFGPTYLDGADVTKIKPHVNH } \\
||:||||||||||||||||||||||||||||||||||||||||||\end{array}$ \\
\hline SARS-CoV-2 & 1 & EVRTIKVFTTVDNINLHTQVVDISMTYGQQF GPTYLDGADVTKIKPHNSH \\
\hline SARS-CoV & 51 & $\begin{array}{l}\text { EGKTFFVLPSDDTLRSEAFEYYHTLDESFLGRYMSALNHTKKWIKFPQVGG } \\
|||||:|||:|||||||||||||\cdot| \cdot||||||||||||||||||||||\end{array}$ \\
\hline SARS-CoV-2 & 51 & EGKTFYVLPNDDTLRVEAFEYYHTTDPSFLGRYMSALNHTKKWKYPQVNG \\
\hline SARS-CoV & 101 & 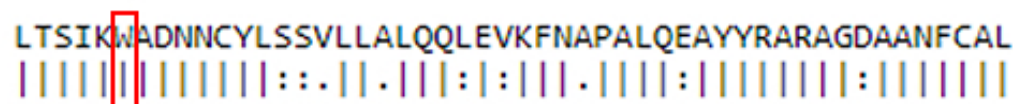 \\
\hline SARS-CoV-2 & 101 & LTSIKWADNCYLATALLTLQQIELKFNPPALQDAYYRARAGEAANFCAL \\
\hline SARS-CoV & 151 & 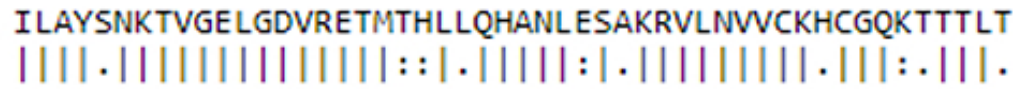 \\
\hline SARS-CoV-2 & 151 & ILAYCNKTVGELGDVRETMSYLFQHANLDSCKRVLNVVCKTCGQQQTTLK \\
\hline SARS-CoV & 201 & 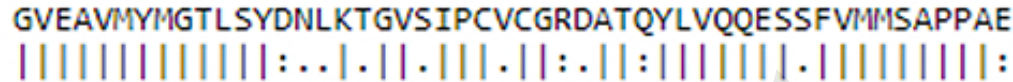 \\
\hline SARS-CoV-2 & 201 & GVEAVMYMGTLSYEQFKKGVQIPCTCGKQATKYLVQQESPFVMIISAPPAQ \\
\hline SARS-CoV & 251 & $\begin{array}{l}\text { YKLQQGTFLCANEYTGNYQCGH YTHITAKETLYRIDGAHLTKMSEYKGPV } \\
|:|:|||| \cdot||:|||||||||||\cdot||:||||| \cdot|||| \cdot|| \cdot||||||:\end{array}$ \\
\hline SARS-CoV-2 & 251 & YELKHGTFTCASEYTGNYQCGHYKHITSKETLYCIDGALLTKSSEYKGPI \\
\hline SARS-COV & 301 & $\begin{array}{l}\text { TDVFYKETSYTTTI--- } \\
\|\|\|\|\|\cdot\|\|\| \|\end{array}$ \\
\hline SARS-CoV-2 & 301 & TDVFYKENSYTTTIKAA \\
\hline
\end{tabular}

Supplementary Figure S4: Pairwise sequence alignment of PLpro derived from SARS-CoV and SARS-CoV-2. The residues inside box are involved in hydrogen bonds and hydrophobic interactions. 